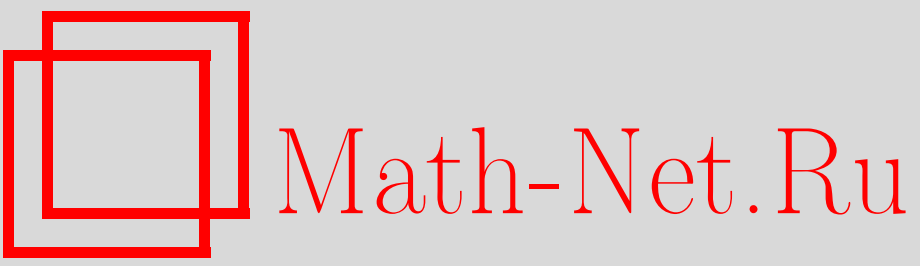

В. К. Белошапка, Вещественные подмногообразия комплексного пространства: их полиномиальные модели, автоморфизмы и проблемы классификации, $У M H$, 2002, том 57, выпуск 1, 3-44

DOI: https://doi.org/10.4213/rm474

Использование Общероссийского математического портала Math-Net.Ru подразумевает, что вы прочитали и согласны с пользовательским соглашением

http://www. mathnet.ru/rus/agreement

Параметры загрузки:

IP : 54.157 .27 .8

26 апреля 2023 г., 10:16:28 


\title{
ВЕЩЕСТВЕННЫЕ ПОДМНОГООБРАЗИЯ КОМПЛЕКСНОГО ПРОСТРАНСТВА: ИХ ПОЛИНОМИАЛЬНЫЕ МОДЕЛИ, АВТОМОРФИЗМЫ И ПРОБЛЕМЫ КЛАССИФИКАЦИИ
}

\author{
В. К. БЕЛОШАПКА
}

Работа представляет собой обзор результатов по локальной теории вещественных подмногообразий комплексного пространства, основная часть которых получена в семинаре А.Г. Витушкина в МГУ за последние 15 лет. Главное достижение это разработка технологии вычисления автоморфизмов, инвариантов и классификаций вещественных подмногообразий, основным этапом которой является построение "хорошей" модельной поверхности. Хорошая модельная поверхность - это аналог соприкасающегося параболоида классической дифференциальной геометрии.

Библиография: 66 названий

\section{СОДЕРЖАНИЕ}

1. Введение

2. Демонстрация метода на примере гиперповерхности в $\mathbb{C}^{2}$

2.1. Выбор модельной поверхности

2.2. Автоморфиизмы модельной поверхности

2.3. Оценка размерности группы автоморфизмов ростка ............... 8

2.4. Что такое хорошая модель $?$.................................. 10

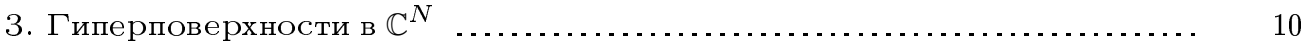

4. Квадратичная модель ростка высокой коразмерности . ............. 13

4.1. Построение модели ...................................... 13

4.2. О классификации квадрик …............................... 17

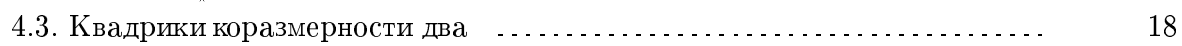

4.4. Феномен жесткости и исключительные квадрики ................ 22

4.5. Вещественные ассоциативные квадрики ........................ 25

4.6. Матричная арифметика Ежова-Шмальца ...................... 26

5. Квадратичные инварианты $C R$-многообразий: три флаговых кон-

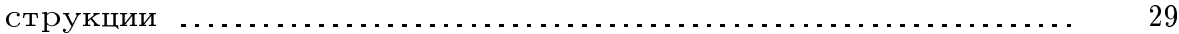

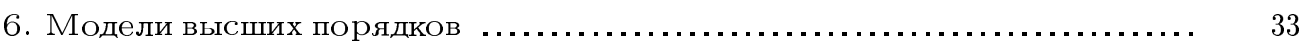

6.1. Кубическая модель ....................................... 34

6.2. Квазипериодическая система модельных поверхностей ............. 37

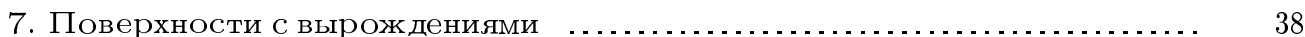

Список литературы .................................................. 41

(C) В.К. БЕЛОШАПКА 2002 


\section{1. Введение}

В 1985 г. вьшел обзор А.Г. Витушкина [64]. Основное содержание обзора - это результаты по невырожденным вещественным гиперповерхностям. Данньй обзор можно рассматривать как продолжение обзора Витушкина. Основное его содержание - это результаты по локальной теории вещественных подмногообразий, полученные участниками семинара за последние 10-15 лет. При этом следует отметить, что, во-первых, в этот период активно изучались многообразия коразмерности более высокой, чем один, а во-вторых, оформилась эффективная технология вычисления автоморфизмов, построения инвариантов и пр., которую мы назьваем методом модельной поверхности.

Данная проблематика представляет интерес не только для многомерного комплексного анализа. Другие разделы математики, питающие интерес к этой области исследования, - это дифференциальная геометрия и уравнения с частными производными. Геометрическая линия - это Э. Картан - Н. Танака - С. Черн и др. [20], [58], [21], [42]. В современной терминологии это техника редукции G-структур. Однако основной, хотя и не единственный, успех метода в применении к вещественным подмногообразиям - это гиперповерхности. Среди работ, в которых данный подход применяется к многообразиям коразмерности выше чем один, кроме классических работ Н. Танаки, упомянем работы Т. Гэррити и Р. Мизнера [33], а также В. Ежова, А. Исаева и Г. Шмальца [25]. Аналитическая линия - это А. Тресс-A. Пуанкаре - Рикье (Riquier) - Ю. Мозер - С. Вебстер - С. Пинчук и др. [59], [47], [36], [21], [65], [46]. Среди современных авторов, тяготеющих к этому, аналитическому, направлению отметим М. Баоуенди, Л. Ротшильд, Н. Стентон, П. Эбенфельда, Д. Зайцева [5], [6], [54], [8], [9], а также А. Сухова [56], [57]. Наш подход, о котором пойдет речь ниже, также примыкает к этой аналитической линии.

Почти все результаты, представленные в данном обзоре, получены единым методом. Ключом к его применению является построение "хорошей модели" (этому термину ниже придается точный смысл). Мы не выделяем из списка результатов главные, а рассматриваем в качестве такого главного результата сам метод модельной поверхности.

Вешественное подмногообразие комплексного пространства - это объект, естественно возникающий в многомерном комплексном анализе. Гиперповерхности - подмногообразия вещественной коразмерности один - это топологические границы областей. Подмногообразия более высокой коразмерности появляются как остовы топологических границ, как их границы Шилова.

Если гладкое вещественное многообразие $M$ поместить в комплексное пространство $\mathbb{C}^{N}$, то между гладкой структурой многообразия и комплексной структурой объемлющего пространства возникает взаимодействие. В результате этого взаимодействия появляются локальные биголоморфные инварианты, группа автоморфизмов ростка многообразия становится конечномерной или просто тривиальной, два наугад выбранных ростка оказьваются неэквивалентньми и т. д. Самые грубые локальные ин-

Работа выполнена при финансовой поддержке Российского фонда фундаментальных исследований (грант № 99-01-00969) и частичной поддержке грантом Шведской академии наук. Работа была начата в Гетеборгском университете, куда автор был любезно приглашен профессором Н. Щербиной. 
варианты - это инварианты, связанные с 1-струей ростка. Первый признак взаимодействия двух упомянутых структур - это появление ненулевой комплексной части в касательном пространстве к многообразию. Если вешественная размерность многообразия достаточно велика, а именно, больше комплексной размерности пространства, то появление комплексной касательной неизбежно. Пару чисел $(n, k)$, где $n-$ размерность комплексной касательной (она может зависеть от точки), а $k$ - коразмерность, будем называть типом многообразия. Тип определяется по 1-струе ростка многообразия в точке. Тип является локальньм биголоморфньм инвариантом. Он не позволяет, например, биголоморфно отобразить комплексную прямую на двумерную вещественную плоскость.

Основное внимание в данной работе уделяется локальным свойствам вещественного подмногообразия комплексного пространства. При этом в центре нашего внимания - три взаимосвязанных цикла вопросов: автоморфизмы, инварианты и классификация.

Для дальнейшего нам понадобятся следующие обозначения. Если $M-$ гладкая поверхность в $\mathbb{C}^{N}, \xi$ - ее точка и $M_{\xi}$ - росток $M$ в точке $\xi$, то через aut $M_{\xi}$ обозначим алгебру Ли ростков вешественных векторных полей с голоморфными коэффициентами в точке $\xi$, касательных к $M_{\xi}$. Если $z=\left(z_{1}, \ldots, z_{N}\right)-$ координаты в $\mathbb{C}^{N}$, то

$$
\text { aut } M_{\xi}=\left\{X(z)=2 \operatorname{Re}\left(f_{1}(z) \frac{\partial}{\partial z_{1}}+\cdots+f_{N}(z) \frac{\partial}{\partial z_{N}}\right)\right\}
$$

где сужение поля $X$ на $M_{\xi}$ есть росток поля, касательного к $M_{\xi}$, а коэффииценты $\left(f_{1}(z), \ldots, f_{n}(z)\right)$ - ростки функций, голоморфных в $\xi$. Это алгебра Ли инфинитезимальных голоморфных автоморфизмов. Эти векторные поля порождают голоморфное действие на $M_{\xi}$. Можно рассмотреть соответствующую aut $M_{\xi}$ локальную группу - Aut $M_{\xi}$. Итак, Aut $M_{\xi}$ - это образ aut $M_{\xi}$ под действием экспоненциального отображения. Эта локальная группа действует на $M_{\xi}$ отображениями, биголоморфными в $\xi$. В дальнейшем эти алгебру и соответствующую ей группу будем называть алгеброй и группой ростка.

\section{2. Демонстрация метода на примере гиперповерхности в $\mathbb{C}^{2}$}

Изучение вешественных многообразий начиналось с изучения многообразий коразмерности один, т.е. гиперповерхностей. Библиография по этой теме есть в обзоре Витушкина [64]. Отметим здесь лишш то, что первая работа по гиперповерхностям это работа А. Пуанкаре 1907 г. [47], в которой изучена самая маломерная ситуация - трехмерная гиперповерхность в $\mathbb{C}^{2}$.

Гиперповерхность в $\mathbb{C}^{2}$ - это поверхность типа $(1,1)$. Продемонстрируем технологию в этой простейшей ситуации.

2.1. Выбор модельной поверхности. Гиперповерхность Г в окрестности своей точки $\xi$-это множество нулей гладкой функции $\rho$, причем $\operatorname{grad} \rho(\xi) \neq 0$. После аффинной замены координат можно считать, что точка $\xi=(0,0), \operatorname{arad} \rho(\xi)=(0, i)$. Пусть $(z, w=u+i v)$ - это координаты в $\mathbb{C}^{2}$, тогда, разрешая уравнение $\rho(z, \bar{z}, u, v)=0$ относительно $v$, получаем уравнение ростка гиперповерхности в нуле в виде $\Gamma_{0}=$ $\{v=F(z, \bar{z}, u)\}$, где функция $F$ и ее первые производные обрашаются в нуль в начале координат. При этом плоскость переменного $z$, т.е. $\{w=0\},-$ это комплексная 
касательная поверхности в нуле, а $\{v=0\}$ - вся касательная плоскость. Если поверхность была вещественно аналитической, то $F$ - это сходяшийся степенной ряд, если только гладкой, то мы вправе рассмотреть формальньй ряд. В пространстве рядов от $(z, \bar{z}, u)$ введем градуировку условиями на веса переменных $[z]=1,[\bar{z}]=1,[u]=2$. Тогда уравнение ростка можно записать в виде $v=2 \operatorname{Re}\left(A z^{2}\right)+h z \bar{z}+\cdots$, где многоточие обозначает члены веса три и вьше. Это уравнение можно переписать в виде $2 \operatorname{Im}\left(w+2 i A z^{2}\right)=h z \bar{z}+\cdots$ или, после простого квадратично-треугольного преобразования координат $z \rightarrow z, w \rightarrow w+2 i A z^{2}$, в виде

$$
v=h z \bar{z}+\cdots \text {. }
$$

Отметим, что одномерная эрмитова форма $h z \bar{z}-$ это форма Леви гиперповерхности $\Gamma_{0}$ в нуле. Если она невырождена (в нашей ситуации это означает, что $h \neq 0$ ), то после замены $z \rightarrow z, w \rightarrow h w$ получаем

$$
v=z \bar{z}+\cdots .
$$

Квадратичная гиперповерхность $Q=\left\{v=|z|^{2}\right\}$ и есть наша модельная поверхность, она же - касательная квадрика.

То, что при выборе модельной поверхности мы не остановились на уровне 1-струи, связано с тем, что все гиперплоскости имеют, как нетрудно убедиться, бесконечномерную групп автоморфизмов. То, что при рассмотрении 2-струи мы ограничились лишь эрмитовьм членом, связано с тем, что от остальных можно избавиться заменой координат и отсечением компонент старшего веса. То, что мы остановились на уровне 2-струи, связано с тем, что уже здесь, как мы увидим ниже, можно получить условие конечномерности групшы ростка.

2.2. Автоморфизмы модельной поверхности. Начнем с вычисления алгебры aut $Q$ - алгебры инфинитезимальных автоморфизмов квадрики $Q$. Записьвая условие касания, получаем следуюшее описание этой алгебры:

$$
\text { aut } Q=\left\{X(z, w)=2 \operatorname{Re}\left(f(z, w) \frac{\partial}{\partial z}+g(z, w) \frac{\partial}{\partial w}\right)\right\},
$$

где голоморфные в окрестности начала координат функции $f$ и $g$ удовлетворяют функциональному уравнению

$$
\operatorname{Im} g\left(z, u+i|z|^{2}\right)=2 \operatorname{Re}\left(f\left(z, u+i|z|^{2}\right) \bar{z}\right) .
$$

Решение этого уравнения проводится в классе формальных степенных рядов с помощью следующей несложной техники. Будем использовать разложения вида

$$
f\left(z, u+i|z|^{2}\right)=\sum_{m=0}^{\infty} \frac{i^{m}}{m !} \Delta^{m} f(z, u)
$$

где $\Delta f=|z|^{2} \frac{\partial}{\partial u} f\left(\Delta^{0}=\mathrm{Id}\right)$, а также

$$
f(z, u)=\sum_{m=0}^{\infty} f_{m}(u) z^{m}
$$


Исходное уравнение будем разлагать на соотношения фиксированных бистепеней по $(z, \bar{z})$. При этом мы получаем бесконечную систему обыкновенных линейных дифоференциальных уравнений на последовательность неизвестных функций $\left(f_{0}(u), g_{0}(u)\right.$, $\left.f_{1}(u), g_{1}(u), \ldots\right)$. Однако, вьписьвая компоненты бистепеней $(2,0),(3,0), \ldots$, убеждаемся, что $g_{2}=g_{3}=\cdots=0$, а компоненты $(3,1),(4,1), \ldots$ дают $f_{3}=f_{4}=\cdots=0$. Если для оставшихся пяти функций $g_{0}, g_{1}, f_{0}, f_{1}, f_{2}$ записать соотношения в компонен$\operatorname{tax}(0,0),(1,0),(1,1),(2,1),(2,2),(3,2),(3,3)$, то, во-первых, оказывается, что решения этой системы обыкновенных линейных уравнений - это многочлены невысоких степеней, во-вторых, оставшийся неиспользованный массив уравнений не дает новых ограничений. В итоге получаем, что алгебра состоит из векторных полей с коэффициентами вида

$$
f=p+\lambda z+a w+2 i \bar{a} z^{2}+r z w, \quad g=q+2 i \bar{p} z+2(\operatorname{Re} \lambda) w+2 i \bar{a} z w+r w^{2},
$$

где $(p, \lambda, a)$ - комплексные параметры, а $(q, r)$ - вещественные и $\operatorname{dim}$ aut $Q=8$.

В алгебре векторных полей введем градуировку условиями:

$[z]=1,[\bar{z}]=1,[w]=2,[\bar{w}]=2,\left[\frac{\partial}{\partial z}\right]=-1,\left[\frac{\partial}{\partial \bar{z}}\right]=-1,\left[\frac{\partial}{\partial w}\right]=-2,\left[\frac{\partial}{\partial \bar{w}}\right]=-2$

Эта градуировка согласована с операцией взятия скобки, т.е. если $g_{m}-$ подпространство полей веса $m$, то $\left[g_{m}, g_{n}\right] \subseteq g_{m+n}$. Из наших вычислений следует, что алгебра aut $Q$ имеет следующую структуру:

$$
\text { aut } Q=g_{-2}+g_{-1}+g_{0}+g_{1}+g_{2}
$$

при этом

$$
\begin{aligned}
g_{-2} & =\left\{2 \operatorname{Re}\left(q \frac{\partial}{\partial w}\right)\right\} \\
g_{-1} & =\left\{2 \operatorname{Re}\left(p \frac{\partial}{\partial z}+2 i \bar{p} z \frac{\partial}{\partial w}\right)\right\} \\
g_{0} & =\left\{2 \operatorname{Re}\left(\lambda z \frac{\partial}{\partial z}+2(\operatorname{Re} \lambda) w \frac{\partial}{\partial w}\right)\right\} \\
g_{1} & =\left\{2 \operatorname{Re}\left(\left(a w+2 i \bar{a} z^{2}\right) \frac{\partial}{\partial z}+2 i \bar{a} z w \frac{\partial}{\partial w}\right)\right\} \\
g_{-2} & =\left\{2 \operatorname{Re}\left(r z w \frac{\partial}{\partial z}+r w^{2} \frac{\partial}{\partial w}\right)\right\}
\end{aligned}
$$

Для того чтобы по алгебре векторных полей получить однопараметрические подгрупшы преобразований, нужно решить соответствующие дифференциальные уравнения. Если $(z, w) \rightarrow(Z(t, z, w), W(t, z, w))$ - искомая подгруппа, соответствующая полю $2 \operatorname{Re}\left(f(z, w) \frac{\partial}{\partial z}+g(z, w) \frac{\partial}{\partial w}\right)$, то функции $(Z, W)$ удовлетворяют системе обцкновенных уравнений с начальными условиями

$$
Z^{\prime}=f(Z, W), \quad W^{\prime}=g(Z, W), \quad Z(0, z, w)=z, \quad W(0, z, w)=w
$$


Решая эти уравнения для полей пяти весовых компонент, получаем

$$
\begin{aligned}
g_{-2} \text { соответствует } z \rightarrow z, w \rightarrow w+t q ; \\
g_{-1} \text { соответствует } z \rightarrow z, w \rightarrow w+2 i \bar{p} z t+i|p|^{2} t^{2} ; \\
g_{0} \text { соответствует } z \rightarrow z \exp (\lambda t), w \rightarrow w \exp (2 \operatorname{Re}(\lambda t)) ; \\
g_{1} \text { соответствует } z \rightarrow \frac{z+a w t}{1-2 i \bar{a} z t-i|a|^{2} w t^{2}}, w \rightarrow \frac{w}{1-2 i \bar{a} z t-i|a|^{2} w t^{2}} ; \\
g_{2} \text { соответствует } z \rightarrow \frac{z}{1-r w t}, w \rightarrow \frac{w}{1-r w t} .
\end{aligned}
$$

Решение систем, соответствуюших весам $-2,-1,0$ и 2 , не вызывает затруднений. Это уравнения с разделяюшимися переменными. Система, соответствующая первой компоненте, решается с помошью следуюшей замены переменных: $Z=1 / \widetilde{Z}, W=\widetilde{W} / \widetilde{Z}$.

Полагая в полученных соотношениях $t=1$, получаем значение экспоненциального отображения на векторах из соответствуюших весовых компонент алгебры. В результате подалгебре $g_{-}=g_{-2}+g_{-1}$ соответствует подгруппа

$$
\text { Aut }_{-} Q=\left\{z \rightarrow z+p, w \rightarrow w+2 i \bar{p} z+\left(q+i|p|^{2}\right)\right\}
$$

действуюшая на $Q$ транзитивно. Тем самым можно констатировать, что $Q$ аффинно-однородна. Подалгебре $g_{0}$ соответствует подгруппа линейных автоморфизмов

$$
\operatorname{Aut}_{0} Q=\left\{z \rightarrow \Lambda z, w \rightarrow|\Lambda|^{2} w\right\}
$$

сохраняющих начало координат. Подалгебре $g_{+}=g_{1}+g_{2}$ соответствует подгрупша

$$
\operatorname{Aut}_{+} Q=\left\{z \rightarrow \frac{z+a w t}{1-\left(2 i \bar{a} z+\left(r+i|a|^{2}\right) w\right)}, w \rightarrow \frac{w}{1-\left(2 i \bar{a} z+\left(r+i|a|^{2}\right) w\right)}\right\}
$$

дробно-линейных автоморфизмов $Q$. Эта подгруппа выделяется условием сохранения начала координат и тем, что якобиева матрица автоморфизма в начале координат имеет на диагонали единицы $(\Lambda=1)$.

2.3. Оценка размерности группы автоморфизмов ростка. То, что модельная поверхность $Q$ играет особую роль по отношению ко всему классу невырожденных гиперповерхностей, убедительнее всего, пожалуй, демонстрирует следующая оценка размерности:

$$
\operatorname{dim} \operatorname{Aut} \Gamma_{\xi} \leqslant \operatorname{dim} \operatorname{Aut} Q
$$

имеющая место для любого невырожденного ростка гладкой гиперповерхности в $\mathbb{C}^{2}$. Приводимое ниже рассуждение представляет собой некую версию теоремы о неявной функции для формальных рядов с оценкой числа параметров.

Теорема о неявной функции утверждает, что нелинейное уравнение имеет единственное решение, коль скоро единственным решением обладает его линейная часть. В нашей ситуации линейной частью нелинейного соотношения окажется уравнение, совпадаюшее с уже известным нам уравнением, определяющим алгебру инфинитезимальных автоморфизмов модельной поверхности $Q$. 
Рассмотрим несколько более общую ситуацию. Пусть отображение вида

$$
f=z+f_{2}+\cdots, \quad g=w+g_{3}+\cdots
$$

переводит росток гиперповерхности

$$
\operatorname{Im} w=|z|^{2}+F(z, \bar{z}, \operatorname{Re} w)
$$

в росток гиперповерхности того же вида

$$
\operatorname{Im} w=|z|^{2}+\widetilde{F}(z, \bar{z}, \operatorname{Re} w)
$$

где компоненты $F$ и $\widetilde{F}$ имеют вес три и вьше. Записывая то, что (4) переводит $(5)$ в (6), мы получим некое тождество. Вот оно:

$$
\begin{aligned}
& \operatorname{Im}\left(w+g_{3}+\cdots\right)=\left|z+f_{2}+\cdots\right|^{2}+\widetilde{F}\left(z+f_{2}+\cdots, \bar{z}+\bar{f}_{2}+\cdots, u+\operatorname{Re} g_{3}+\cdots\right), \\
& \text { где } w=u+i\left(|z|^{2}+\widetilde{F}(z, \bar{z}, \operatorname{Re} w)\right) . \\
& \text { Отделяя в этом соотношении компоненту веса }(m+1), \text { получаем }
\end{aligned}
$$

$$
\operatorname{Re}\left(i g_{m+1}+2 f_{m} \bar{z}\right)=F_{m+1}-\widetilde{F}_{m+1}+\cdots
$$

где в левой части $w=u+i|z|^{2}$, а многоточие в правой части - это члены, зависящие от $f_{j}, g_{j+1}, F_{j+1}, \widetilde{F}_{j+1}$ при $j<m$.

При фиксированных $F$ и $\widetilde{F}$ эти соотношения позволяют рекуррентно вычислять полиномиальные компоненты отображения $\left(f_{m}, g_{m+1}\right)$, решая алгебраическую систему линейных уравнений, правая часть которой зависит от компонент, вычисленных ранее. Размерность множества решений неоднородной системы линейных уравнений не превьшает размерности пространства решений однородной. Но однородные уравнения - это в точности уравнения (3), определяющие алгебру aut $Q$. Поэтому число свободных параметров, задающих отображение в классе формальных степенных рядов указанного вида, не превосходит размерности подалгебры aut $Q$, которая в данной ситуации равна трем (параметры $a$ и $r$ ). И, тем самым, число параметров, задаюших произвольное отображение этих ростков, не превосходит размерности всей алгебры aut $Q$, т.е. восьми. Применяя полученную оценку к отображению ростка в себя, получаем нашу оценку на размерность группы автоморфизмов ростка.

Отметим, что значение проведенного вьгисления не сводится только к оценке размерности. Получено большее - указана система параметров, однозначно определяющих отображения одного невырожденного ростка в другой такой росток. В частности, получена параметризация автоморфизмов произвольного ростка. Действительно, рассмотрим систему параметров $(p, q, \Lambda, a, r)$, определяющую автоморфизм гиперквадрики $Q$ и представляюшую собой часть 2-струи этого автоморфизма. Эта система параметров позволяет однозначно определить отображение ростка в росток, при этом эти параметры могут быть связанными дополнительными соотношениями (см. [10]), которые приводят к падению размерности. В общем положении эта размерность равна нулю. 
2.4. Что такое хорошая модель? Перечислим основные этапы проведенного исследования.

1. Выбор хорошей модельной поверхности.

2. Вычисление алгебры и группы модельной поверхности.

3. Параметризация отображений ростков алгеброй модельной поверхности.

Сформулируем еше раз свойства хорошей модельной поверхности $Q$.

1. Универсальность: росток любой невырожденной гиперповерхности в $\mathbb{C}^{2}$ эквивалентен ростку вида (2).

2. Конечномерность: (а) группа голоморфных автоморфизмов $Q$ конечномерна; (b) любая гиперповерхность, заданная уравнениями степени меньшей, чем два, имеет бесконечномерную группу.

3. Однородность: гиперквадрика $Q$ однородна, т.е. ее голоморфные автоморфизмы действуют на ней транзитивно, в данной ситуации однородность обеспечивается аффинными автоморфизмами.

4. Симметричность: (а) гиперквадрика является самой симметричной невырожденной гиперповерхностью, т.е. размерность группы ростка невырожденной гиперповерхности не превосходит размерности группы касательной гиперквадрики; (b) алгебра модельной поверхности параметризует семейство отображений одного невырожденного ростка в другой.

5. Алгебраические свойства: (а) алгебра Ли голоморфных векторных полей на невырожденной гиперквадрике - это некоторая алгебра полиномиальных векторных полей ограниченной степени, в данной ситуации степени коэффициентов не превосходят двойки; (b) группа автоморфизмов невырожденной гиперквадрики - это группа Ли, являющаяся подгрупой группы дробно-линейных преобразований $\mathbb{C}^{2}$.

В дальнейшем, когда мы будем утверждать, что некая поверхность является хорошей модельной поверхностью для ростков того или иного типа, мы будем стремиться получить для нее, в той или иной редакции, этот список свойств.

Есть свойства, специфические для данной ситуации, - поверхности типа $(1,1)$. Отметим два из них.

(1) Модельная поверхность единственна, т.е. одна и та же квадрика $Q$ является хорошей моделью любого невырожденного ростка типа $(1,1)$.

(2) Модельная поверхность является дробно-линейным образом стандартной сфферы. Действительно, замена

$$
\widetilde{z}=\frac{z}{w-i}, \quad \widetilde{w}=\frac{i(w+i)}{w-i}
$$

переводит $Q=\left\{\operatorname{Im} \widetilde{w}=|\widetilde{z}|^{2}\right\}$ в $\left\{|z|^{2}+|w|^{2}=1\right\}$. При этом точке $(0, i)$ соответствует пересечение $Q$ с проективной бесконечностью.

\section{3. Гиперповерхности в $\mathbb{C}^{N}$}

Невырожденные гиперповерхности в пространстве произвольной размерности изучались в ряде работ (см. [58], [21], [64]).

Рассуждения, аналогичные проведенным для $\mathbb{C}^{2}$, показьвают, что уравнение ростка гладкой гиперповерхности в пространстве $\mathbb{C}^{n+1}$ с координатами $\left(z=\left(z_{1}, \ldots, z_{n}\right)\right.$, 
$w=u+i v)$ можно записать в виде

$$
v=\langle z, \bar{z}\rangle+\cdots
$$

где $\langle z, \bar{z}\rangle$ - эрмитова форма (форма Леви), точками обозначены члены веса три и вьше (веса вычисляем как и раньше: $[z]=1,[\bar{z}]=1,[u]=2$ ). Модельной поверхностью в этой ситуации является гиперквадрика $Q=\{v=\langle z, \bar{z}\rangle\}$. По отношению к гиперповерхности (7) квадрику $Q$ будем называть касательной квадрикой. Условие конечномерности группы Aut $Q$ - это условие невырожденности эрмитовой формы $\langle z, \bar{z}\rangle$. Если форма невырождена, то невырожденными называем росток гиперповерхности и квадрику. Если форма вырождена, то после ее приведения к сумме квадратов одна из переменных, пусть $z_{j}$, будет отсутствовать. Тогда любое преобразование вида $z_{j} \rightarrow f\left(z_{j}\right)$, где $f^{\prime}(0) \neq 0$ (остальные переменные на месте) является автоморфизмом и группа Aut $Q$ - бесконечномерна. Если же форма невырождена, то $Q$ является хорошей модельной поверхностью, т.е. имеет место типовой пакет утверждений:

1. Универсальность: росток любой гиперповерхности в $\mathbb{C}^{n+1}$ эквивалентен ростку вида (7).

2. Конечномерность: (а) группа голоморфных автоморфизмов гиперквадрики общего положения конечномерна; (b) критерием конечномерности групш голоморфных автоморфизмов $Q$ является невырожденность формы $\langle z, \bar{z}\rangle$; (c) любая гиперповерхность, заданная уравнениями степени меньшей, чем два, имеет бесконечномерную групшу.

3. Однородность: гиперквадрика $Q$ однородна, т.е. ее голоморфные автоморфизмы действуют на ней транзитивно, однородность обеспечивается аффинными автоморфизмами.

4. Симметричность: (а) гиперквадрика является самой симметричной невырожденной гиперповерхностью, т.е. размерность группы ростка невырожденной гиперповерхности не превосходит размерности группы касательной гиперквадрики; (b) алгебра модельной поверхности параметризует семейство отображений одного невырожденного ростка в другой.

5. Алгебраические свойства: (а) алгебра Ли голоморфных векторных полей на невырожденной гиперквадрике - это некоторая алгебра полиномиальных векторных полей ограниченной степени, в данной ситуации степени коэффициентов не превосходят двойки; (b) группа автоморфизмов невырожденной гиперквадрики - это группа Ли, являющаяся подгруппй группы дробно-линейных преобразований $\mathbb{C}^{n+1}$.

Алгебра aut $Q$ имеет следующую структуру (градуировка та же): aut $Q=g_{-2}+$ $g_{-1}+g_{0}+g_{1}+g_{2}$, при этом компоненты имеют следующее явное описание:

$$
\begin{aligned}
g_{-2} & =\left\{2 \operatorname{Re}\left(q \frac{\partial}{\partial w}\right)\right\}, \\
g_{-1} & =\left\{2 \operatorname{Re}\left(p \frac{\partial}{\partial z}+2 i\langle z, \bar{p}\rangle \frac{\partial}{\partial w}\right)\right\}, \\
g_{0} & =\left\{2 \operatorname{Re}\left(\lambda z \frac{\partial}{\partial z}+\rho w \frac{\partial}{\partial w}\right)\right\}, \\
g_{1} & =\left\{2 \operatorname{Re}\left((a w+2 i\langle z, \bar{a}\rangle z) \frac{\partial}{\partial z}+2 i\langle z, \bar{a}\rangle w \frac{\partial}{\partial w}\right)\right\},
\end{aligned}
$$




$$
g_{2}=\left\{2 \operatorname{Re}\left(r w z \frac{\partial}{\partial z}+r w^{2} \frac{\partial}{\partial w}\right)\right\}
$$

где $q, r \in \mathbb{R}, p, a \in \mathbb{C}^{n},(n \times n)$-матрица $\lambda$ и вещественное число $\rho$ связаны соотношением $2 \operatorname{Re}\langle\lambda z, \bar{z}\rangle=\rho\langle z, \bar{z}\rangle$. Подалгебре $g_{-}=g_{-2}+g_{-1}$ соответствует подгруппа

$$
\text { Aut }_{-} Q=\{z \rightarrow z+p, w \rightarrow w+2 i\langle z, \bar{p}\rangle+(q+i\langle p, \bar{p}\rangle)\}
$$

действуюшая на $Q$ транзитивно. Эта подгрупша известна как группа Гейзенберга. Подалгебре $g_{0}+g_{1}+g_{2}$ соответствует подгруппа автоморфизмов $Q$, сохраняющих неподвижным начало координат (подгруппа стабильности). Подалгебра $g_{0}$ порождает в этой подгрупе подгруппу $\operatorname{Aut}_{0} Q$ линейных автоморфизмов, которая является связной компонентой групш LAut $Q$ всех линейных автоморфизмов $Q$, $\operatorname{coxpаняющих~}$ начало координат. Чтобы получить описание этой групшы, рассмотрим более общую ситуацию. Пусть отображение вида

$$
f=\Lambda z+f_{2}+\cdots, \quad g=\rho w+g_{3}+\cdots
$$

переводит росток гиперповерхности

$$
\operatorname{Im} w=\langle z, \bar{z}\rangle_{1}+F_{1}(z, \bar{z}, \operatorname{Re} w)
$$

в росток гиперповерхности того же вида

$$
\operatorname{Im} w=\langle z, \bar{z}\rangle_{2}+F_{2}(z, \bar{z}, \operatorname{Re} w)
$$

где компоненты $F_{1}$ и $F_{2}$ имеют вес три и выше. Записьвая в виде тождества то, что (8) переводит (9) в (10), и отделяя в этом тождестве компоненты веса один и два, получаем, что линейная часть отображения имеет вид

$$
\left(\begin{array}{ll}
\Lambda & * \\
0 & \rho
\end{array}\right),
$$

причем $\langle\Lambda z, \overline{\Lambda z}\rangle_{2}=\rho\langle z, \bar{z}\rangle_{1}$. Из этого вычисления есть несколько следствий:

(1) действие голоморфных отображений на форму Леви сводится к действию группы $G L(n, \mathbb{C}) \oplus \mathbb{R}^{*}$ по формуле $\langle z, \bar{z}\rangle \rightarrow \rho\left\langle\Lambda^{-1} z, \overline{\Lambda^{-1} z}\right\rangle ;$

$(2)$ две модельные гиперповерхности $Q_{1}$ и $Q_{2}$ голоморфно эквивалентны тогда и только тогда, когда они эквивалентны линейно;

(3) группа линейных автоморфизмов $Q$, сохраняющих начало координат, LAut $Q$ имеет вид $z \rightarrow \Lambda z, w \rightarrow \rho w$, где $\langle\Lambda z, \overline{\Lambda z}\rangle=\rho\langle z, \bar{z}\rangle$; алгебра $g_{0}$ является алгеброй этой групшы.

Алгебре $g_{+}=g_{1}+g_{2}$ соответствует групп Aut $+Q$, которую составляют нелинейные автоморфизмы $Q$. Это автоморфизмы, сохраняюшие начало координат, линейная часть которых имеет следуюший блочный вид:

$$
\left(\begin{array}{cc}
\operatorname{Id}_{n} & * \\
0 & 1
\end{array}\right)
$$




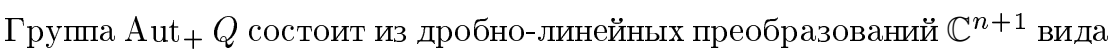

$$
\begin{gathered}
z \rightarrow \frac{z+a w}{1-(2 i\langle z, \bar{a}\rangle+(r+i\langle a, \bar{a}\rangle) w)}, \\
w \rightarrow \frac{w}{1-(2 i\langle z, \bar{a}\rangle+(r+i\langle a, \bar{a}\rangle) w)} .
\end{gathered}
$$

Вьпишем размерности:

$\operatorname{dim} g_{-2}=\operatorname{dim} g_{2}=1, \quad \operatorname{dim} g_{-1}=\operatorname{dim} g_{1}=2 n$, $\operatorname{dim}$ LAut $Q=\operatorname{dim} g_{0}=n^{2}+1, \quad \operatorname{dim}_{\text {Aut }} Q=\operatorname{dim}_{-} \mathrm{Aut}_{+} Q=2 n+1$,

$$
\operatorname{dim} \operatorname{Aut} Q=(n+2)^{2}-1 \text {. }
$$

При $n>1$ имеется конечное множество попарно неэквивалентных модельных гиперповерхностей. Если $(\mu, \nu)$ - сигнатура невырожденной формы, то единственным инвариантом является $|\mu-\nu|$. Модельная гиперповерхность эквивалентна гиперсфере лиш в случае знакоопределенной формы. При $n=2$ имеем две неэквивалентных модели $v=\left|z_{1}\right|^{2}+\left|z_{2}\right|^{2}$ и $v=\left|z_{1}\right|^{2}-\left|z_{2}\right|^{2}$. Несмотря на наличие неэквивалентных гиперквадрик, размерности групп одинаковы для всех классов.

Гиперквадрика не является единственной однородной гиперповерхностью в комплексном пространстве. Список таких гиперповерхностей в пространстве $\mathbb{C}^{2}$ был получен Э. Картаном в 1932 г. [20]. Этот список наряду с отдельными гиперповерхностями содержит семейства, зависяшие от одного вешественного параметра. Список замечателен также тем, что все гиперповерхности заданы как линии уровня элементарных функций.

Серия публикаций А.В. Лободы (см. [40] и др.) направлена на построение классификации однородных гиперповерхностей в $\mathbb{C}^{3}$. Построен обширный список. Работа, по-видимому, близка к завершению. Список Лободы обладает свойствами списка Картана: конечномерность пространства модулей (двумерные семейства) и представимость элементарными функциями.

\section{4. Квадратичная модель ростка высокой коразмерности}

4.1. Построение модели. Пусть теперь $M_{\xi}$ - росток гладкой поверхности произвольной коразмерности $k \geqslant 1$ в $\mathbb{C}^{N}$, т.е. совокупность общих нулей ростков нескольких гладких функций, обрашаюшихся в нуль в точке $\xi$, т.е. $M_{\xi}=\left\{\zeta \in \mathbb{C}^{N}: \rho_{1}(\zeta)=\cdots=\right.$ $\left.\rho_{k}(\zeta)=0\right\}$. Гладкость ростка гарантируется условием линейной независимости градиентов $\left(\operatorname{grad} \rho_{1}(\xi), \ldots, \operatorname{grad} \rho_{k}(\xi)\right)$ как векторов пространства $\mathbb{R}^{2 N}$. Однако уже на уровне 1-струи может иметь место некое соотношение, от которого мы хотим освободиться. Потребуем, чтобы эти градиенты были комплексно линейно независимы, т.е. линейно независимы над полем комплексных чисел. В этом случае росток назьвается порождающим, причем если $n$ - размерность комплексной касательной, то $n+k=N$ и вешественная размерность ростка есть $2 n+k$.

Итак, пусть $M_{\xi}$ - росток порождающей поверхности в $\mathbb{C}^{n+k}$ типа $(n, k)$, где $n \geqslant 1$, $k \geqslant 1$. Выбирая подходящим образом координаты $\left(z=\left(z_{1}, \ldots, z_{n}\right), w=\left(w_{1}, \ldots, w_{k}\right)\right)$, уравнения ростка можно записать так: $\operatorname{Im} w=F(z, \bar{z}, \operatorname{Re} w)$, где у $F$ отсутствуют постоянный и линейные члены. А вводя веса координат и осуществляя квадратичное 
преобразование, по аналогии с тем, что мы делали для гиперповерхности, получаем уравнение ростка в виде

$$
\operatorname{Im} w=\langle z, \bar{z}\rangle+O(3) .
$$

Поверхность $Q=\{\operatorname{Im} w=\langle z, \bar{z}\rangle\}$ по аналогии с гиперповерхностью будем назьвать касательной квадрикой ростка $M_{\xi}$. Основное отличие от случая гиперповерхности состоит в том, что эрмитова форма $\langle z, \bar{z}\rangle=\left(\langle z, \bar{z}\rangle_{1}, \ldots,\langle z, \bar{z}\rangle_{k}\right)$ принимает векторные значения.

Прежде всего в этой новой ситуации встает вопрос о критерии конечномерности групшы автоморфизмов квадрики. Есть две очевидные причины потери конечномерности. Во-первых, форма может иметь ненулевое ядро, т.е. найдется вектор $e \neq 0$ такой, что $\langle e, \bar{z}\rangle=0$ для всех $z \in \mathbb{C}^{n}$. Сделаем в $\mathbb{C}^{n}$ линейную замену, которая переводит $е$ в первьй вектор стандартного базиса $(1,0, \ldots, 0)$. После этого форма не будет содержать переменное $z_{1}$ ни в одной из координат. Теперь любая замена $z_{1} \rightarrow f\left(z_{1}\right)$, где $f^{\prime}(0) \neq 0$ (остальные переменные на месте), является автоморфизмом и группа Aut $Q$ бесконечномерна. Во-вторых, координатные эрмитовы формы могут оказаться линейно зависимыми. Пусть, например, $\langle z, \bar{z}\rangle_{1}$ есть линейная комбинация остальных форм, тогда после подходящей линейной замены переменной $w$ форма $\langle z, \bar{z}\rangle_{1}$ становится равной нулю. Теперь замена $g_{1} \rightarrow g\left(w_{1}\right)$ (остальные переменные на месте), где $g-$ любой локально обратимый степенной ряд с вешественньми коэффициентами, является автоморфизмом и группа Aut $Q$ опять бесконечномерна. Оказьвается, что других причин для бесконечномерности нет, и это является основанием для того, чтобы принять эти два условия в качестве определения невырожденности векторнозначной эрмитовой формы [11].

ОПРЕДЕЛЕНИЕ 1 . Говорим, что эрмитова форма $\langle z, \bar{z}\rangle=\left(\langle z, \bar{z}\rangle_{1}, \ldots,\langle z, \bar{z}\rangle_{k}\right)$ и соответствующая ей квадрика невырождены, если

(a) из $\langle e, \bar{z}\rangle=0$ для всех $z$ следует, что $e=0$,

(b) формы $\left(\langle z, \bar{z}\rangle_{1}, \ldots,\langle z, \bar{z}\rangle_{k}\right)$ линейно независимы.

При $k=1$ условие (а) означает обычную невырожденность, а условие (b) есть следствие (a). Отметим сразу, что условие (b) невьполнимо при $k>n^{2}$, т.е. невырожденность квадрики - это условие, которое является условием общего положения только в диапазоне коразмерностей $k=1, \ldots, n^{2}$.

Оказьвается, что квадрика типа $(n, k)$ при $k \leqslant n^{2}$ является хорошей моделью ростка того же типа, а именно ([11], [12]) справедливы следуюшие утверждения.

1. Универсальность: любой порождаюший росток типа $(n, k)$ в $\mathbb{C}^{n+k}$ эквивалентен ростку вида (11).

2. Конечномерность: (а) группа голоморфных автоморфизмов квадрики общего положения является конечномерной группой Ли; (b) критерием конечномерности группыголоморфных автоморфизмов $Q$ является невырожденность формы $\langle z, \bar{z}\rangle$ (в смысле определения 1); (с) любая поверхность, заданная уравнениями степени меньшей, чем два, имеет бесконечномерную групу.

3. Однородность: квадрика $Q$ однородна, т.е. ее голоморфные автоморфизмы действуют на ней транзитивно, однородность обеспечивается аффинными автоморфизмами. 
4. Симметричность: (а) квадрика является самой симметричной невырожденной поверхностью, т.е. размерность групы ростка невырожденной поверхности не превосходит размерности групшы ее касательной квадрики; (b) алгебра автоморфизмов квадрики параметризует семейство отображений одного невырожденного ростка в другой.

5. Алгебраические свойства: (а) алгебра Ли голоморфных векторных полей на невырожденной квадрике - это некоторая алгебра полиномиальных векторных полей ограниченной степени, степени коэффициентов не превосходят двойки; (b) группа автоморфизмов невырожденной квадрики - это группа Ли, являюшаяся подгруппй групшы бирациональных преобразований $\mathbb{C}^{n+k}$, чьи степени равномерно по всей группе ограничены [60], в качестве оценки степени числителей и знаменателей в несократимом представлении можно взять $4(n+k)$ [13]; (c) если два ростка эквивалентны, то эквивалентны их касательные квадрики, две квадрики голоморфно эквивалентны в том и только том случае, когда они эквивалентны линейно.

Алгебра квадрики сохраняет прежнюю структуру (градуировка та же): aut $Q=$ $g_{-2}+g_{-1}+g_{0}+g_{1}+g_{2}$, при этом компоненты имеют следующее явное описание:

$$
\begin{gathered}
g_{-2}=\left\{2 \operatorname{Re}\left(q \frac{\partial}{\partial w}\right)\right\}, \text { где } q \in \mathbb{R}^{k} ; \\
g_{-1}=\left\{2 \operatorname{Re}\left(p \frac{\partial}{\partial z}+2 i\langle z, \bar{p}\rangle \frac{\partial}{\partial w}\right)\right\}, \text { где } p \in \mathbb{C}^{n} ; \\
g_{0}=\left\{2 \operatorname{Re}\left(\lambda z \frac{\partial}{\partial z}+\rho w \frac{\partial}{\partial w}\right)\right\},
\end{gathered}
$$

где $\lambda$ и $\rho$ - квадратные матрицы размерностей $n$ и $k$, связанные соотношением $2 \operatorname{Re}\langle\lambda z, \bar{z}\rangle=\rho\langle z, \bar{z}\rangle$

$$
g_{1}=\left\{2 \operatorname{Re}\left((a w+A(z, z)) \frac{\partial}{\partial z}+2 i\langle z, \bar{a} w\rangle \frac{\partial}{\partial w}\right)\right\},
$$

где $A$ - квадратичная форма со значениями в $\mathbb{C}^{n}, a-$ линейное отображение из $\mathbb{C}^{k}$ в $\mathbb{C}^{n}$, связанные соотношением $\langle A(z, z), \bar{z}\rangle=2 i\langle z, \bar{a}\langle z, \bar{z}\rangle\rangle$;

$$
g_{2}=\left\{2 \operatorname{Re}\left((B(z, w)) \frac{\partial}{\partial z}+r(w, w) \frac{\partial}{\partial w}\right)\right\},
$$

где $B$ - билинейная форма со значениями в $\mathbb{C}^{n}, r$-квадратичная форма со значениями в $\mathbb{C}^{k}$, связанные соотношениями $\operatorname{Re}\langle B(z, u), \bar{z}\rangle=r(\langle z, \bar{z}\rangle, u), \operatorname{Im}\langle B(z,\langle z, \bar{z}\rangle), \bar{z}\rangle=0$.

Группа, соответствующая $g_{-}=g_{-2}+g_{-1}$, вполне аналогична той, что была при $k=1$. Это группа Aut_ $Q$, состояшая из афффинных преобразований вида

$$
\{z \rightarrow z+p, w \rightarrow w+2 i\langle z, \bar{p}\rangle+(q+i\langle p, \bar{p}\rangle)\}
$$

транзитивно действуюшая на $Q$ (аналог группы Гейзенберга).

Нетрудно показать, что подгруппа линейных автоморфизмов LAut $Q$ состоит из преобразований $z \rightarrow \Lambda z, w \rightarrow \rho w$, где $\Lambda \in G L(n, \mathbb{C}), \rho \in G L(k, \mathbb{R})$, причем $\langle\Lambda z, \overline{\Lambda z}\rangle=$ $\rho\langle z, \bar{z}\rangle$. Алгебра $g_{0}$ является алгеброй этой групшы. 
Подалгебре $g_{+}=g_{1}+g_{2}$ соответствует подгруппа Aut $Q$ преобразований $Q$ вида $z \rightarrow z+f_{2}+\cdots, w \rightarrow w+g_{3}+\cdots-$ подгрупп нелинейных автоморфизмов, сохраняющих начало координат. Столь простого и явного описания этой подгруппы, как при $k=1$, нет. Результаты, касаюшиеся ее строения, будут обсуждаться ниже, однако имеет место некое обобщение дробно-линейности, а именно бирациональность + ограниченность степени (теорема Туманова), которую мы включили в общий список под номером 5.(b).

Центральньм утверждением данного списка является то, что алгебра невырожденной квадрики состоит из полей с квадратичньми коэффициентами. При $k=1-$ это следствие основной леммы из первой части работы Мозера и Черна [21]. При $k>1$ конструкция, вполне аналогичная мозеровской, позволяет свести решение основного уравнения (условие касания) в формальных рядах к решению системы линейных дифоференциальных уравнений с постоянными коэффициентами. Но в отличие от случая гиперповерхности, где уравнения были обыкновенньми, это будет система в частных производных. В получении критерия конечномерности основную роль играет теорема об экспоненциальном представлении решения линейной, с постоянньми коэффициентами системы дифференциальных уравнений с частными производными (Мальгранжа-Паламодова-Эренпрайса). В соответствии с этой теоремой критерием конечномерности пространства решений системы является условие, что характеристическое множество является конечным набором точек. Для системы уравнений, к которой сводится условие касания, невырожденность эрмитовой формы означает, что характеристическое множество состоит только из начала координат. Это одновременно дает полиномиальность решений. Дополнительные рассуждения позволяют доказать, что степени решений, в действительности, не превосходят двух [11], [12].

Пункт 5.(c) нашего списка является следствием того, что действие голоморфных отображений на ростках поверхностей порождает линейное действие на касательных квадриках. Действительно, пусть имеется два эквивалентных ростка

$$
\begin{aligned}
& M_{1}=\left\{\operatorname{Im} w=\langle z, \bar{z}\rangle_{1}+\cdots\right\} \text { и } \\
& M_{2}=\left\{\operatorname{Im} w=\langle z, \bar{z}\rangle_{2}+\cdots\right\}
\end{aligned}
$$

и $z \rightarrow f(z, w), w \rightarrow g(z, w)$ - отображение первого во второй. Тогда из младших компонент возникающего в этой ситуации соотношения нетрудно получить, что $f=\Lambda z+f_{2}+\cdots, g=\rho w_{2}+g_{3}+\cdots$, причем $\langle\Lambda z, \overline{\Lambda z}\rangle_{2}=\rho\langle z, \bar{z}\rangle_{1}$. Отсюда можно сделать два вьвода. Во-первых, если $M_{1}$ и $M_{2}$ это квадрики, то линейное отображение $z \rightarrow \Lambda z, w \rightarrow \rho w$ отображает первую квадрику на вторую. А во-вторых, действие голоморфного отображения на росток поверхности порождает следующее действие групшы $G L(n, \mathbb{C}) \oplus G L(k, \mathbb{R})$ на форму $\langle z, \bar{z}\rangle:\langle z, \bar{z}\rangle \rightarrow \rho\left\langle\Lambda^{-1} z, \overline{\Lambda^{-1} z}\right\rangle$. A это в свою очередь означает, что все инварианты формы по отношению к этому действию оказьваются инвариантами ростка по отношению к биголоморфным преобразованиям. К построению этих инвариантов мы вернемся ниже.

Форма Леви имеет внутреннее определение (см., например, [22]) через коммутатор векторных полей. После выбора дополнения комплексной касательной до всего касательного пространства эта форма становится эрмитовой формой на комплексной касательной со значениями в этом дополнении, совпадающей с формой $\langle z, \bar{z}\rangle$ - формой Леви ростка в отмеченной точке. Это определение можно распространить на аб- 
страктно определенные $C R$-многообразия. При этом если два ростка $C R$-многообразий $C R$-эквивалентны, то их формы Леви связаны посредством вьшеуказанного действия $G L(n, \mathbb{C}) \oplus G L(k, \mathbb{R})$. Поэтому любые инварианты векторной эрмитовой формы относительно этого действия автоматически являются $C R$-инвариантами $C R$-многообразия.

Условие невырожденности является самым слабым условием, гарантируюшим конечномерность. Есть и другие вполне естественные условия, из которых условие невырожденности следует.

Так, квадрика назьвается сильно невырожденной, если координатные формы $\langle z, \bar{z}\rangle=\left(\langle z, \bar{z}\rangle_{1}, \ldots,\langle z, \bar{z}\rangle_{k}\right)$ линейно независимы и из них можно составить невырожденную, в обычном, скалярном смысле, линейную комбинацию. Для квадрик типа $(2,2)$ оба определения эквивалентны. Самая маломерная ситуация, где они различаются, это тип $(3,2)$. Вот простейший пример невырожденной квадрики, не являющейся сильно невырожденной:

$$
H_{1}=\left(\begin{array}{lll}
1 & 1 & 0 \\
1 & 0 & 0 \\
0 & 0 & 0
\end{array}\right), \quad H_{2}=\left(\begin{array}{lll}
1 & 0 & 1 \\
0 & 0 & 0 \\
1 & 0 & 0
\end{array}\right) \text {. }
$$

Сильно невырожденная квадрика назьвается положительно определенной, если среди линейных комбинаций координатных форм есть положительно определенная. Положительно определенные квадрики - это остовы (границы Шилова) областей Зигеля второго рода. Области Зигеля играют большую роль в теории однородных ограниченных областей и автоморфных функций [34], [48], [42].

$\mathrm{C}$ каждой векторнозначной эрмитовой формой $\langle z, \bar{z}\rangle$ можно связать конус $C=$ $\operatorname{int}\left(\operatorname{conv}\left(\left\{\langle z, \bar{z}\rangle: z \in \mathbb{C}^{n}\right\}\right)\right)$, т.е. множество внутренних точек выпуклой оболочки образапространства $\mathbb{C}^{n}$ под действием отображения, осуществляемого формой. Если конус $C$ непустой и острый, то соответствующая область Зигеля

$$
D=\left\{(z, w) \in \mathbb{C}^{n} \oplus \mathbb{C}^{k}: \operatorname{Im} w-\langle z, \bar{z}\rangle \in C\right\}
$$

является однородной областью, биголоморфно эквивалентной ограниченной.

Область $D$ при этом является оболочкой голоморфности квадрики $Q=\{(z, w) \in$ $\left.\mathbb{C}^{n} \oplus \mathbb{C}^{k}: \operatorname{Im} w=\langle z, \bar{z}\rangle\right\}[42]$. Таким образом, положительно определенные квадрики являются объектом, общим для обеих теорий: как для теории однородных ограниченных областей, так и для рассматриваемой нами теории хороших моделей. Ряд обсуждаемых здесь результатов для частного случая положительно определенных квадрик были получены как результаты об областях Зигеля и их остовах. Это касается конечномерности группы автоморфизмов такой квадрики, бирациональности ее автоморфизмов, описания структуры алгебры ее инфинитезимальных автоморфизмов [50].

4.2. О классификации квадрик. Как было отмечено вьше, действие голоморфных отображений на ростках типа $(n, k)$ порождает следующее действие групшы $G L(n, \mathbb{C}) \oplus G L(k, \mathbb{R})$ на форму $\langle z, \bar{z}\rangle:\langle z, \bar{z}\rangle \rightarrow \rho\left\langle\Lambda^{-1} z, \overline{\Lambda^{-1} z}\right\rangle$. Если же представлять форму набором эрмитовых матриц $\left(H_{1}, \ldots, H_{k}\right)$, то это действие вида $(\Lambda, \rho)\left(H_{1}, \ldots, H_{k}\right)=\rho\left(\left(\Lambda^{*}\right)^{-1} H_{1} \Lambda^{-1}, \ldots,\left(\Lambda^{*}\right)^{-1} H_{k} \Lambda^{-1}\right) . \quad$ Есть простой способ, позволяюший редуцировать это действие к действию групшы $G L(n, \mathbb{C})$. Нужно 
перейти от записи квадрики как набора из $k$ линейно независимых векторов вешественного линейного пространства эрмитовых матриц к сопоставлению квадрике $k$-мерной плоскости в этом пространстве. Таким образом, если не различать квадрики, отличаюшиеся линейной заменой переменной $w$, то множество квадрик типа $(n, k)$ отождествляется с вещественньм грассмановьм многообразием $G r_{k}\left(\mathbb{R}^{n^{2}}\right)$. В таком представлении действие сводится к действию $G L(n, \mathbb{C})$ на этом грассмановом многообразии. Классы эквивалентных квадрик - это орбиты данного действия. Линейная подгруппа группы автоморфизмов некоторой квадрики соответствует подгрупше в $G L(n, \mathbb{C})$, сохраняюшая соответствуюшую $k$-мерную плоскость.

Классификация квадрик произвольного типа по отношению к этому действию представляется весьма необозримой задачей. Однако для некоторых типов квадрик, а именно для $k=1,2, n^{2}-2, n^{2}-1, n^{2}$, эта задача имеет хороший ответ. Число классов эквивалентности невырожденных квадрик типа $(n, 1)$ конечно и равно целой части числа $\frac{n+2}{2}$. Все невырожденные квадрики типа $\left(n, n^{2}\right)$ эквивалентны, так как каждая из них представлена базисным набором из $n^{2}$ матриц, а любые два базиса связаны линейньм преобразованием. Таким образом, имеется лишь один класс таких квадрик. Задача классификации квадрик коразмерности два былаполностью решена в работах С. Шевченко [52], [53].

4.3. Квадрики коразмерности два. Квадрики коразмерности два - это тип $(n, 2)$, следующий за гиперквадриками. Квадрика типа $(n, 2)$ задается парой эрмитовых матрищ $H_{1}$ и $H_{2}$, т.е. $Q=\left\{\operatorname{Im} w_{1}=H_{1} z \cdot \bar{z}, \operatorname{Im} w_{2}=H_{2} z \cdot \bar{z}\right\}$. Классификация пары эрмитовых форм с точностью до линейных замен переменного $z$ - это одна из классических задач линейной алгебры. Общеизвестна возможность одновременного приведения пары форм к диагональному виду при условии, что одна из форм положительно определена. Довольно известной является теорема о каноническом виде пары форм при условии, что одна из форм невырождена [35]. Однако даже для классификации невырожденных квадрик этого недостаточно, так как есть невырожденные квадрики коразмерности два такие, что все линейные комбинации координатных форм вырождены. Поэтому классификационная теорема требуется в полном объеме [62]. На основе этой классификации и используя дополнительную возможность, связанную с возможностью перехода к линейной комбинации форм, Шевченко строит полную классификацию квадрик коразмерности два.

Каждой паре эрмитовых матриц $\left(H_{1}, H_{2}\right)$ сопоставляется пучок матриц $\left(t_{1} H_{1}+\right.$ $\left.t_{1} H_{2}\right)$, его характеристический многочлен $\operatorname{det}\left(t_{1} H_{1}+t_{2} H_{2}\right)$, где $\left(t_{1}: t_{2}\right) \in \mathbb{C P}^{1}$, совокупность корней этого многочлена, а также некоторьй набор целочисленных параметров: минимальные индексы и множители инерции (см. [62]). Для того чтобы две пары эрмитовых форм были эквивалентны посредством замены переменного $z$, необходимо и достаточно совпадение следуюшего набора данных: корней, минимальных индексов и множителей инерции. Изучение действия $G L(2, \mathbb{R})$ (замены переменной $w$ ) на этом множестве данных позволяет дать критерий эквивалентности двух квадрик. Он заключается в том, что первый набор корней переводится во второй дробно-линейным преобразованием с вещественными коэффициентами, и в вьполнении некоторых соотношений между минимальными индексами и множителями инерции первой и второй пары.

Канонический вид пары матриц имеет блочно-диагональную форму (наподобие 
жордановой нормальной формы), где на диагоналях стоят пары клеток трех видов: первый соответствует вещественному корню и имеет в качестве своих параметров кроме самого корня размер клетки и множитель инерции, второй соответствует паре комплексно сопряженных корней и определяется корнем и размером клетки (четное число), третий соответствует корню на бесконечности и определяется только размером клетки (нечетное число). Размеры клеток первого и второго видов определяются элементарными делителями пучка, а третьего - минимальными индексами. Канонический вид сильно невырожденных квадрик содержит лишш клетки первых двух типов.

Характеристический многочлен имеет в общем положении $n$ различных корней. Система из $n$ различных упорядоченных точек на комплексной плоскости при $n \geqslant 4$ по отношению к групе дробно-линейных преобразований описывается системой из $n-3$ числовых инвариантов (двойные отношения), эта совокупность параметров вместе с дискретными инвариантами задает класс эквивалентности квадрик коразмерности два. Поэтому при $n \leqslant 3$ число классов эквивалентности квадрик типа $(n, 2)$ конечно: при $n=2$ их три, при $n=3$ - десять.

Описание алгебры инфинитезимальных автоморфизмов квадрики, приведенное выше, является явным с точностью до получения значений параметров $(p, q, \Lambda, \rho, a, A, B, r)$ из определяюших соотношений (см. п. 4.1). Для фиксированной квадрики эти соотношения являются системой линейных алгебраических уравнений на неизвестные значения параметров. Коэффициенты этих уравнений зависят от элементов эрмитовых матриц, задаюших форму $\langle z, \bar{z}\rangle$. Эта зависимость почти во всех соотношениях линейная и лишш в одном - квадратичная. Полученная для коразмерности два классификация позволяет дать явное описание пространства решений этой системы и ответить на ряд качественных вопросов. Прежде всего это вопросы о размерностях градуированных компонент алгебры aut $Q=g_{-2}+g_{-1}+g_{0}+g_{1}+g_{2}$. Параметры $p$ и $q$, задающие компоненты $g_{-2}$ и $g_{-1}$, не связаны никакими соотношениями, соответственно для любой квадрики коразмерности два $\operatorname{dim} g_{-2}=2$ и $\operatorname{dim} g_{-1}=2 n$. В [52] приводятся описание $g_{0}, g_{1}, g_{2}$ и вычисление их размерностей через инварианты пучка $t_{1} H_{1}+t_{2} H_{2}$. Сформулируем некоторые результаты этой работы.

1. Пусть квадрика сильно невырожденная. Можно считать, что $\operatorname{det} H_{1} \neq 0$, положим $H=\left(H_{1}\right)^{-1} H_{2}$. Возможны три случая.

1.1. Если матрица $H$ диагонализуема (заменами переменной $z$ ) и имеет два различных собственных значения, то $\operatorname{dim} g_{1}=2 n, \operatorname{dim} g_{2}=2$.

1.2. Если $H$ имеет единственное собственное значение $\lambda$ и $(H-\lambda E)^{2}=0$, то также $\operatorname{dim} g_{1}=2 n, \operatorname{dim} g_{2}=2$.

1.3. В остальных случаях $\operatorname{dim} g_{1}=0, \operatorname{dim} g_{2}=0$.

2. Если невырожденная квадрика не является сильно невырожденной, то $\operatorname{dim} g_{1}<$ $2 n, \operatorname{dim} g_{2}=2$.

3.1. Если $n=2$, то $4 \leqslant \operatorname{dim} g_{0} \leqslant 5$.

3.2. Если $n \geqslant 3$, то $n+1 \leqslant \operatorname{dim} g_{0} \leqslant(n-1)^{2}+4$.

Таким образом:

$$
\begin{array}{ll}
\text { при } n=2 & 10 \leqslant \operatorname{dim} g_{0}+\operatorname{dim} g_{1}+\operatorname{dim} g_{2} \leqslant 11, \\
\text { при } n \geqslant 3 & n+1 \leqslant \operatorname{dim} g_{0}+\operatorname{dim} g_{1}+\operatorname{dim} g_{2} \leqslant n^{2}+7 .
\end{array}
$$


Любая невырожденная квадрика типа $(2,2)$ эквивалентна одной из трех квадрик: $Q_{1}, Q_{-1}$ или $Q_{0}$.

$$
Q_{1}=\left\{\operatorname{Im} w_{1}=\left|z_{1}\right|^{2}+\left|z_{2}\right|^{2}, \operatorname{Im} w_{2}=z_{1} \bar{z}_{2}+z_{2} \bar{z}_{1}\right\}
$$

Характеристический многочлен $Q_{1}$ имеет два вещественных корня. Данная квадрика является прямым произведением двух квадрик типа $(1,1)$ и в других координатах может быть записана в виде $Q_{1}=\left\{\operatorname{Im} w_{1}=\left|z_{1}\right|^{2}, \operatorname{Im} w_{2}=\left|z_{2}\right|^{2}\right\}$. Вот данные о размерностях компонент алгебры: $\operatorname{dim} g_{0}=4, \operatorname{dim} g_{1}=4, \operatorname{dim} g_{2}=2$. Отметим, что эта квадрика положительно определенная, т.е. является каноническим видом любой положительно определенной квадрики типа $(2,2)$.

$$
Q_{-1}=\left\{\operatorname{Im} w_{1}=\left|z_{1}\right|^{2}-\left|z_{2}\right|^{2}, \operatorname{Im} w_{2}=z_{1} \bar{z}_{2}+z_{2} \bar{z}_{1}\right\}
$$

Характеристический многочлен $Q_{-1}$ имеет два комплексно-сопряженных корня. Размерности компонент алгебры - те же, что и у $Q_{1}$.

$$
Q_{0}=\left\{\operatorname{Im} w_{1}=\left|z_{1}\right|^{2}, \operatorname{Im} w_{2}=z_{1} \bar{z}_{2}+z_{2} \bar{z}_{1}\right\}
$$

Характеристический многочлен $Q_{0}$ имеет один кратный корень, $\operatorname{dim} g_{0}=5$, $\operatorname{dim} g_{1}=4, \operatorname{dim} g_{2}=2$.

Ежов и Шмальц предложили называть квадрику $Q_{1}$ гиперболической, $Q_{-1}$ эллиптической и $Q_{0}$ параболической. В 8-мерном вешественном линейном пространстве квадрик типа $(2,2)$ квадрики, эквивалентные $Q_{1}$ и $Q_{-1}$, лежат по разные стороны от конической гиперповерхности второго порядка, на которой располагаются квадрики, эквивалентные $Q_{0}$. Все три квадрики являются сильно невырожденными, т.е. всякая невырожденная квадрика типа $(2,2)$ является сильно невырожденной [39], [12], [26].

Каждая квадрика типа $(3,2)$ эквивалентна одной и только одной квадрике из следуюшего списка из десяти позиций. Данные по размерностям компонент $g_{0}, g_{1}, g_{2}$ приводим в виде строки $\left(d_{0}+d_{1}+d_{2}=d\right)$.

Квадрики $Q_{1}$ и $Q_{2}$ - это случай трех различных вещественных собственных значений, что для квадрики означает диагонализуемость (одновременное приведение к сумме квадратов). При этом $Q_{1}$ положительно определена.

$$
\begin{aligned}
& Q_{1}=\left\{\operatorname{Im} w_{1}=\left|z_{1}\right|^{2}+\left|z_{2}\right|^{2}+\left|z_{3}\right|^{2}, \operatorname{Im} w_{2}=\left|z_{2}\right|^{2}-\left|z_{3}\right|^{2}\right\} \quad(4+0+0=4), \\
& Q_{2}=\left\{\operatorname{Im} w_{1}=-\left|z_{1}\right|^{2}+\left|z_{2}\right|^{2}+\left|z_{3}\right|^{2}, \operatorname{Im} w_{2}=\left|z_{2}\right|^{2}-\left|z_{3}\right|^{2}\right\} \quad(4+0+0=4) .
\end{aligned}
$$

Следующие четыре квадрики - случай двух вещественных собственных значений. У $Q_{3}$ и $Q_{4}$ все клетки в каноническом виде первого порядка, обе квадрики приводимы (прямое произведение двух гиперквадрик), причем $Q_{3}$ положительно определена, а $Q_{4}$ - нет. $Q_{5}$ и $Q_{6}$ в каноническом виде имеют клетку второго порядка.

$$
\begin{aligned}
& Q_{3}=\left\{\operatorname{Im} w_{1}=\left|z_{1}\right|^{2}+\left|z_{2}\right|^{2}, \operatorname{Im} w_{2}=\left|z_{3}\right|^{2}\right\} \quad(7+6+2=15), \\
& Q_{4}=\left\{\operatorname{Im} w_{1}=-\left|z_{1}\right|^{2}+\left|z_{2}\right|^{2}, \operatorname{Im} w_{2}=\left|z_{3}\right|^{2}\right\} \quad(7+6+2=15), \\
& Q_{5}=\left\{\operatorname{Im} w_{1}=\left|z_{1}\right|^{2}+2 \operatorname{Re}\left(z_{2} \bar{z}_{3}\right), \operatorname{Im} w_{2}=\left|z_{1}\right|^{2}+\left|z_{2}\right|^{2}\right\} \quad(6+0+0=6), \\
& Q_{6}=\left\{\operatorname{Im} w_{1}=-\left|z_{1}\right|^{2}+2 \operatorname{Re}\left(z_{2} \bar{z}_{3}\right), \operatorname{Im} w_{2}=-\left|z_{1}\right|^{2}+\left|z_{2}\right|^{2}\right\} \quad(6+0+0=6) .
\end{aligned}
$$


Следующие две квадрики - это единственное собственное значение. $Q_{7}$ - это случай клеток первого и второго порядков, $Q_{8}-$ клетка третьего порядка.

$$
\begin{aligned}
& Q_{7}=\left\{\operatorname{Im} w_{1}=\left|z_{1}\right|^{2}+2 \operatorname{Re}\left(z_{2} \bar{z}_{3}\right), \operatorname{Im} w_{2}=\left|z_{2}\right|^{2}\right\} \quad(8+6+2=16), \\
& Q_{8}=\left\{\operatorname{Im} w_{1}=\left|z_{2}\right|^{2}+2 \operatorname{Re}\left(z_{1} \bar{z}_{3}\right), \operatorname{Im} w_{2}=2 \operatorname{Re}\left(z_{1} \bar{z}_{2}\right)\right\} \quad(6+0+0=6) .
\end{aligned}
$$

Следуюшая квадрика - это случай одного вешественного и пары комплексно сопряженных собственных значений:

$$
Q_{9}=\left\{\operatorname{Im} w_{1}=\left|z_{1}\right|^{2}+2 \operatorname{Re}\left(z_{2} \bar{z}_{3}\right), \operatorname{Im} w_{2}=2 \operatorname{Im}\left(z_{2} \bar{z}_{3}\right)\right\} \quad(4+0+0=4) .
$$

Последняя, десятая квадрика - это невырожденная квадрика, не являюшаяся сильно невырожденной (нуль-квадрика). В каноническом виде ей соответствует клетка третьего вида.

$$
Q_{10}=\left\{\operatorname{Im} w_{1}=2 \operatorname{Re}\left(z_{1} \bar{z}_{3}\right), \operatorname{Im} w_{2}=2 \operatorname{Re}\left(z_{2} \bar{z}_{3}\right)\right\} \quad(8+2+0=10) .
$$

Размерность пространства решений системы линейных уравнений определяется ее рангом. Поскольку коэффициенты системы зависят от коэффициентов эрмитовых форм не более чем квадратично, то стратификация квадрик по рангамградуированных компонент дается полиномиальными соотношениями (равенство нулю миноров). Поэтому вне собственного алгебраического подмножества в пространстве квадрик (прямая сумма $k$ экземпляров пространства эрмитовых матриц) все размерности будут находиться на своей нижней гранище. Таким образом, квадрика типа $(3,2)$ общего положения - это либо $Q_{1}$, либо $Q_{2}$, либо $Q_{9}$. Все три случая - это три различных собственных значения. В первых двух они вещественны, в третьем есть пара комплексно-сопряженных. Первьй, в отличие от второго, положительно определенный.

Для квадрики общего положения типа $(n, 2)$ при $n \geqslant 3$ размерности компонент следуюшие: $\operatorname{dim} g_{0}=n+1, \operatorname{dim} g_{1}=\operatorname{dim} g_{2}=0$. То, что эта нижняя оценка достигается и, тем самым, реализуется для квадрики обшего положения, показывает любая диагонализуемая квадрика с различньми корнями характеристического многочлена, например,

$$
\left\{\operatorname{Im} w_{1}=\left|z_{1}\right|^{2}+\left|z_{2}\right|^{2}+\cdots+\left|z_{n}\right|^{2}, \operatorname{Im} w_{2}=1\left|z_{1}\right|^{2}+2\left|z_{2}\right|^{2}+\cdots+n\left|z_{n}\right|^{2}\right\} .
$$

Квадрика типа $(n, 2), n \geqslant 2$, на которой достигаются все верхние оценки размерностей компонент (см. выше), - это

$$
\left\{\operatorname{Im} w_{1}=2 \operatorname{Re}\left(z_{1} \bar{z}_{2}\right)+\left|z_{3}\right|^{2}+\cdots+\left|z_{n}\right|^{2}, \operatorname{Im} w_{2}=\left|z_{1}\right|^{2}\right\} .
$$

Компоненты имеют следуюшие размерности: $\operatorname{dim} g_{-2}=\operatorname{dim} g_{2}=2, \operatorname{dim} g_{-1}=$ $\operatorname{dim} g_{1}=2 n, \operatorname{dim} g_{0}=(n-1)^{2}+4$. Отметим, что это суммарно на единицу больше (из-за $g_{0}$ ), чем размерность алгебры приводимой, т.е. распадаюшейся в прямое произведение, квадрики

$$
\left\{\operatorname{Im} w_{1}=\left|z_{2}\right|^{2}+\left|z_{3}\right|^{2}+\cdots+\left|z_{n}\right|^{2}, \operatorname{Im} w_{2}=\left|z_{1}\right|^{2}\right\}
$$

Полученное описание алгебры автоморфизмов позволило Ежову и Шмальцу доказать, что любой автоморфизм невырожденной $(n, 2)$-квадрики реализуется рациональным отображением, чья степень не превосходит двух [26]. Напомним, что для гиперквадрик автоморфизмы - это дробно-линейные преобразования. Для сильно невырожденных квадрик коразмерности два этот результат ранее был получен Абросимовым [1]. Его работа основана на другой технике и независима от классификации Шевченко. 
4.4. Феномен жесткости и исключительные квадрики. Случай коразмерности один обладает особенностью, которая довольно редко встречается в более высоких коразмерностях. Малая деформация невырожденной гиперквадрики в классе гиперквадрик дает гиперквадрику, эквивалентную исходной. Это обстоятельство позволяет применять к гиперповерхностям технологию Э. Картана и методы теории $G$-структур [55], [58], [21; часть II], [61]. Есть еще два особых случая, обладающих тем же свойством, это две последних (для невырожденных квадрик) коразмерности $k=n^{2}-1$ и $k=n^{2}$. Причем эти случаи являются особыми еше в одном отношении: любая такая квадрика обладает нетривиальной нелинейной подгруппой Aut $+Q$. Оказьвается, что при $2 \leqslant k \leqslant n^{2}-2$ у квадрики общего положения (т.е. вне некоторого собственного алгебраического множества) нелинейных автоморфизмов нет. Более того, при $3 \leqslant k \leqslant n^{2}-3$ линейная подгруппа автоморфизмов квадрики обшего положения LAut $Q$ тривиальна в следуюшем смысле. Эта подгруппа состоит лишь из скалярных растяжений $\left\{z \rightarrow \lambda z, w \rightarrow|\lambda|^{2} w, \lambda \in \mathbb{C}^{*}\right\}$ $[12],[45],[30]$. Такие квадрики называются жесткими. При этом отсутствие нелинейных автоморфизмов называем $N$-жесткостью, а отсутствие нетривиальных линейных автоморфизмов $L$-жесткостью, т.е. жесткость - это одновременное наличие обоих условий. Можно говорить отдельно о $g_{0}$-жесткости $\left(\operatorname{dim} g_{0}=2\right), g_{1}$-жесткости $\left(\operatorname{dim} g_{1}=0\right)$ и $g_{2}$-жесткости $\left(\operatorname{dim} g_{2}=0\right)$. Между этими условиями имеются связи, например $g_{2}$-жесткость следует как из $g_{0}$-жесткости [12], так и из $g_{1}$-жесткости [44]. Группа автоморфизмов жесткой квадрики состоит из афффинных преобразований, составляюших группу Aut_ $Q$, которая одинакова у всех квадрик данного типа, и скалярных растяжений. Ситуащия $k=2$, как и $k=n^{2}-2$, требует оговорок, так как здесь подгруппа линейных автоморфизмов квадрики обшего положения имеет размерность $(n+1)$ и не сводится, тем самым, к скалярньм растяжениям.

Наличие некоторой симметрии между квадриками типа $(n, k)$ и $\left(n, n^{2}-k\right)$ имеет свою причину. Квадрика коразмерности $k$ - это набор из $k$ линейно независимых эрмитовых матриц. Если не различать квадрики, связанные линейной заменой переменного $w$, то это $k$-мерная плоскость в $n^{2}$-мерном вещественном линейном пространстве эрмитовых матриц $\mathscr{H}$. У этой плоскости есть двойственное описание - описание в виде совокупности общих нулей независимого набора из $n^{2}-k$ линейных форм на $\mathscr{H}$. Фиксируя изоморфизм между $\mathscr{H}$ и $\mathscr{H}^{*}$, мы получаем соответствие между квадриками типа $(n, k)$ и $\left(n, n^{2}-k\right)$. При этом линейные подгрупшы LAut $Q$, соответствующие двойственным квадрикам, оказываются изоморфными.

Жесткость квадрики общего положения в диапазоне коразмерностей от 3 до $n^{2}-3$ оставляет возможность для сушествования исключительных квадрик с нелинейными автоморфизмами и нетривиальной линейной подгруппой. Есть простой способ строить такие квадрики: это прямое произведение квадрик. Если сомножители невырождены, то алгебра произведения есть прямая сумма алгебр сомножителей и групша соответственно есть прямое произведение групп автоморфизмов сомножителей [12]. Если сомножители имели нетривиальные группы, то нетривиальна будет и группа произведения. В списке квадрик типа $(3,2)$ такими приводимыми квадриками являются $Q_{3}$ и $Q_{4}$. Размерности подгрупп, сохраняюших начало координат, для обеих квадрик равны 15. Причина появления богатых групп автоморфизмов для этих квадрик ясна. Размерность складывается из размерностей групшы гиперквадрики типа $(2,1)-$ 10 и типа $(1,1)-5$. Однако в списке имеется неприводимая квадрика $Q_{7}$ с подгруп- 
пой стабильности размерности 16. Таким образом, задача классификации квадрик с нелинейными автоморфизмами и поиска самой симметричной невырожденной квадрики, т.е. квадрики, чья группа имеет максимальную для данного типа размерность, является вполне содержательной. Для квадрик коразмерности два эта задача решается на основе классификации Шевченко (см. выше).

Для квадрик типа $(3,3)$, т.е. квадрик коразмерности три в $\mathbb{C}^{6}$, эта задача была решена Н. Палинчак [43], [44]. Любая $(3,3)$-квадрика, обладающая нелинейными автоморфизмами, эквивалентна одной из следующих восьми квадрик. Данные по размерностям компонент приводим в прежнем формате: $\operatorname{dim} g_{0}+\operatorname{dim} g_{1}+\operatorname{dim} g_{2}$. Напомним, что $\operatorname{dim} g_{-2}+\operatorname{dim} g_{-1}=3+6=9$ для всех квадрик этого типа и что $\operatorname{dim} Q=2 n+k=9$.

$$
\begin{array}{ll}
Q_{1}: & \operatorname{Im} w_{1}=\left|z_{1}\right|^{2}, \quad \operatorname{Im} w_{2}=\left|z_{2}\right|^{2}, \quad \operatorname{Im} w_{3}=\left|z_{3}\right|^{2}, \\
Q_{2}: & \operatorname{Im} w_{1}=\left|z_{1}\right|^{2}-\left|z_{2}\right|^{2}, \quad \operatorname{Im} w_{2}=2 \operatorname{Re}\left(z_{1} \bar{z}_{2}\right), \quad \operatorname{Im} w_{3}=\left|z_{3}\right|^{2}, \\
Q_{3}: & \operatorname{Im} w_{1}=\left|z_{1}\right|^{2}, \quad \operatorname{Im} w_{2}=2 \operatorname{Re}\left(z_{1} \bar{z}_{2}\right), \quad \operatorname{Im} w_{3}=\left|z_{3}\right|^{2} .
\end{array}
$$

Первые три квадрики - это прямое произведение одной $(2,2)$-квадрики (список см. вьше) и единственной $(1,1)$-квадрики. Поскольку первая $(2,2)$-квадрика списка есть прямое произведение двух $(1,1)$-квадрик, то $Q_{1}$ является прямым произведением трех $(1,1)$-квадрик. Вот размерности групп: $Q_{1}-(6+6+3=15), Q_{2}-(6+6+3=15)$, $Q_{3}-(7+6+3=16)$;

$$
\begin{array}{ll}
Q_{4}: \quad \operatorname{Im} w_{1}=\left|z_{1}\right|^{2}, \quad \operatorname{Im} w_{2}=2 \operatorname{Re}\left(z_{1} \bar{z}_{2}\right), \quad \operatorname{Im} w_{3}=2 \operatorname{Re}\left(z_{1} \bar{z}_{3}\right)+\left|z_{2}\right|^{2} \\
\quad(8+6+3=17) ; \\
Q_{5}: \quad \operatorname{Im} w_{1}=2 \operatorname{Re}\left(z_{1} \bar{z}_{3}\right), \quad \operatorname{Im} w_{2}=2 \operatorname{Re}\left(z_{2} \bar{z}_{3}\right), \quad \operatorname{Im} w_{3}=2 \operatorname{Im}\left(z_{1} \bar{z}_{2}\right) \\
\quad(10+6+3=19) ; \\
Q_{6}: \quad \operatorname{Im} w_{1}=2 \operatorname{Re}\left(z_{1} \bar{z}_{3}\right), \quad \operatorname{Im} w_{2}=2 \operatorname{Re}\left(z_{2} \bar{z}_{3}\right), \quad \operatorname{Im} w_{3}=\left|z_{3}\right|^{2} \\
\quad(10+6+3=19) ; \\
Q_{7}: \quad \operatorname{Im} w_{1}=2 \operatorname{Re}\left(z_{1} \bar{z}_{3}\right), \quad \operatorname{Im} w_{2}=2 \operatorname{Re}\left(z_{2} \bar{z}_{3}\right), \quad \operatorname{Im} w_{3}=2 \operatorname{Im}\left(z_{2} \bar{z}_{3}\right) \\
\quad(8+6+3=17) ; \quad \operatorname{Im} w_{2}=2 \operatorname{Im}\left(z_{1} \bar{z}_{2}\right), \quad \operatorname{Im} w_{3}=2 \operatorname{Re}\left(z_{1} \bar{z}_{3}\right)+\left|z_{2}\right|^{2} \\
Q_{8}: \quad \operatorname{Im} w_{1}=2 \operatorname{Re}\left(z_{1} \bar{z}_{2}\right), \quad \\
\quad(7+2+1=10) .
\end{array}
$$

Напомним, что $(3,3)$-квадрика общего положения является жесткой, т.е. $(2+0+$ $0=2)$. В приведенном списке нет квадрик, чья группа автоморфизмов линейна, но нетривиальна ( $\left.\operatorname{dim} g_{0}>2\right)$. Однако самая грубая оценка показьвает, что $\operatorname{dim} g_{0} \leqslant 18$. Поэтому можно утверждать, что размерность группы любой $(3,3)$-квадрики не превосходит 19, и если размерность группы некоторой квадрики равна 19, то квадрика эквивалентна либо $Q_{5}$, либо $Q_{6}$. В работе А. Арбатского [4] для каждой из квадрик списка вычислена группа автоморфизмов. Все автоморфизмы реализуются бирациональньми преобразованиями $\mathbb{C}^{6}$, чьи степени не превосходят трех.

$(3,4)$-квадрика общего положения является жесткой, т.е. имеет структуру $(2+$ $0+0)$. Классификация квадрик типа $(3,4)$, т.е. квадрик коразмерности 4 в $\mathbb{C}^{7}$, с нелинейными автоморфизмами была построена Е. Анисовой [2], [3]. Любая такая 
квадрика эквивалентна одной из девяти попарно неэквивалентных квадрик данного списка:

$$
\begin{aligned}
& Q_{1}: \quad \operatorname{Im} w_{1}=2 \operatorname{Re}\left(z_{1} \bar{z}_{3}\right), \quad \operatorname{Im} w_{2}=2 \operatorname{Re}\left(z_{2} \bar{z}_{3}\right), \\
& \operatorname{Im} w_{3}=2 \operatorname{Im}\left(z_{1} \bar{z}_{3}\right), \quad \operatorname{Im} w_{4}=2 \operatorname{Im}\left(z_{2} \bar{z}_{3}\right) \quad(10+6+4=20) ; \\
& Q_{2}: \quad \operatorname{Im} w_{1}=2 \operatorname{Re}\left(z_{1} \bar{z}_{3}\right), \quad \operatorname{Im} w_{2}=2 \operatorname{Re}\left(z_{2} \bar{z}_{3}\right), \\
& \operatorname{Im} w_{3}=\left|z_{3}\right|^{2}, \quad \operatorname{Im} w_{4}=2 \operatorname{Im}\left(z_{2} \bar{z}_{3}\right) \quad(11+6+4=21) ; \\
& Q_{3}: \quad \operatorname{Im} w_{1}=2 \operatorname{Re}\left(z_{1} \bar{z}_{3}\right), \quad \operatorname{Im} w_{2}=2 \operatorname{Re}\left(z_{1} \bar{z}_{2}\right) \text {, } \\
& \operatorname{Im} w_{3}=\left|z_{2}\right|^{2}, \quad \operatorname{Im} w_{4}=\left|z_{1}\right|^{2} \quad(8+2+1=11) \text {; } \\
& Q_{4}: \quad \operatorname{Im} w_{1}=2 \operatorname{Re}\left(z_{1} \bar{z}_{2}\right), \quad \operatorname{Im} w_{2}=-2 \operatorname{Im}\left(z_{1} \bar{z}_{2}\right) \text {, } \\
& \operatorname{Im} w_{3}=2 \operatorname{Re}\left(z_{1} \bar{z}_{3}\right), \quad \operatorname{Im} w_{4}=\left|z_{2}\right|^{2} \quad(8+2+1=11) ; \\
& Q_{5}: \quad \operatorname{Im} w_{1}=2 \operatorname{Re}\left(z_{1} \bar{z}_{2}\right), \quad \operatorname{Im} w_{2}=-2 \operatorname{Im}\left(z_{1} \bar{z}_{2}\right) \text {, } \\
& \operatorname{Im} w_{3}=2 \operatorname{Re}\left(z_{1} \bar{z}_{3}\right), \quad \operatorname{Im} w_{4}=\left|z_{1}\right|^{2}+\left|z_{2}\right|^{2} \quad(7+2+1=10) ; \\
& Q_{6}: \quad \operatorname{Im} w_{1}=2 \operatorname{Re}\left(z_{1} \bar{z}_{2}\right), \quad \operatorname{Im} w_{2}=-2 \operatorname{Im}\left(z_{1} \bar{z}_{2}\right) \text {, } \\
& \operatorname{Im} w_{3}=\left|z_{1}\right|^{2}, \quad \operatorname{Im} w_{4}=\left|z_{3}\right|^{2} \quad(10+4+4=18) ; \\
& Q_{7}: \quad \operatorname{Im} w_{1}=2 \operatorname{Re}\left(z_{1} \bar{z}_{2}\right), \quad \operatorname{Im} w_{2}=-2 \operatorname{Im}\left(z_{1} \bar{z}_{2}\right) \text {, } \\
& \operatorname{Im} w_{3}=\left|z_{1}\right|^{2}, \quad \operatorname{Im} w_{4}=2 \operatorname{Re}\left(z_{1} \bar{z}_{3}\right)+\left|z_{2}\right|^{2} \quad(8+4+2=14) ; \\
& Q_{8}: \quad \operatorname{Im} w_{1}=2 \operatorname{Re}\left(z_{1} \bar{z}_{2}\right), \quad \operatorname{Im} w_{2}=\left|z_{1}\right|^{2} \text {, } \\
& \operatorname{Im} w_{3}=\left|z_{2}\right|^{2}, \quad \operatorname{Im} w_{4}=\left|z_{3}\right|^{2} \quad(7+2+2=11) ; \\
& Q_{9}: \quad \operatorname{Im} w_{1}=2 \operatorname{Re}\left(z_{1} \bar{z}_{2}\right), \quad \operatorname{Im} w_{2}=2 \operatorname{Im}\left(z_{1} \bar{z}_{2}\right) \text {, } \\
& \operatorname{Im} w_{3}=\left|z_{1}\right|^{2}-\left|z_{2}\right|^{2}, \quad \operatorname{Im} w_{4}=\left|z_{3}\right|^{2} \quad(7+2+1=10) \text {. }
\end{aligned}
$$

Следует отметить, что первые две квадрики не являются сильно невырожденными, при этом вторая из них $Q_{2}$ является самой симметричной квадрикой типа $(3,4)$. Среди этих квадрик присутствуют приводимые квадрики - прямые произведения различных $(2,3)$-квадрик на единственную гиперквадрику типа $(1,1)$, это $Q_{6}, Q_{8}$ и $Q_{9}$.

Как классификация Палинчак, так и классификация Анисовой построены на основе классификации Шевченко пар эрмитовых матриц третьего порядка. Обе классификации для доказательства попарной неэквивалентности полученных квадрик кроме размерностей компонент алгебр используют квадратичные инварианты, построенные в [13], [14], которые мы обсудим ниже.

Как видно из приведенных классификаций, во всех перечисленных случаях имеет место любопытная симметрия: $\operatorname{dim} g_{j} \leqslant \operatorname{dim} g_{-j}, j=1,2$, причем верхняя граница достижима. В связи в этим, в качестве гипотезы, предполагалось, что это верно для квадрик всех типов. Однако недавно П. Уткиным [63] было показано, что для $(5,3)$-квадрик эта симметрия может нарушаться. Этот пример обобшается на $n \geqslant 5$, $k \geqslant 3$.

С исключительными квадриками, имеющими нелинейные автоморфизмы связан интересный вопрос об оценке их степени. В соответствии с теоремой Туманова [60] группа автоморфизмов - это группа Ли, действующая на квадрике бирациональными преобразованиями $\mathbb{C}^{n+k}$ ограниченной степени. Используя то, что алгебра автоморфизмов состоит из полей, чьи степени не превосходят двух, можно доказать, что 
степени автоморфизмов не превосходят $4(n+k)$. Однако, несмотря на обилие изученных ситуаций, никто не смог предъявить квадрики, чьи автоморфизмы имеют степень больше $k$. Квадрик, для которых степень равна коразмерности, достаточно много: они есть в списках Шевченко, Палинчак и Анисовой, такие квадрики легко конструируются как прямое произведение гиперквадрик, такую же степень имеют автоморфизмы однородных областей Зигеля II-го рода. Но общее утверждение остается пока лишш в виде гипотезы.

ГИПОТЕЗА О СТЕПЕНИ. Группа автоморфизмов невырожденной квадрики типа $(n, k)$ действует бирациональными преобразованиями $\mathbb{C}^{n+k}$, чьи степени (числителей и знаменателя в несократимой записи) не превосходят коразмерности, т.е. $k$.

Тот тип груп, которые возникают здесь в качестве груп автоморфизмов невырожденных квадрик, достоин внимания. Это подгрупшы группы бирациональных преобразований комплексного линейного пространства (группы Кремоны), состоящие из преобразований ограниченной степени. Если подставить рациональное преобразование в себя, то степень композиции увеличится. Это рассуждение, конечно, требует “общности положения", и наличие наших групп это и есть те примеры, которые его опровергают. Есть пример, где причина того, что степень при подстановке не возрастает, очевидна. Это пример проективных преобразований. Поэтому естественно стремление объяснить существование таких подгрупп с помощью сведения их к проективньм преобразованиям. В этом отношении представляет интерес следующая теорема (Д. Зайцев [66]):

Пусть $G$ - вешественная связная группа Ли, действуюшая в $\mathbb{C}^{n}$ бирациональными преобразованиями, голоморфньми в некоторой области (одной для всей группы). Тогда существует линейное представление $G$ на некотором $\mathbb{C}^{N+1}$ и бирациональное $G$-эквивариантное отображение из $\mathbb{C}^{n}$ на $\mathbb{C P}^{N}$.

Условие того, что автоморфизмы области продолжаются до бирациональных преобразований объемлющего пространства, приведено в работе Вебстера [65]. Для груп автоморфизмов квадрик теорема Зайцева не применима, но это не означает, само по себе, что не справедливо ее заключение. Отметим, забегая вперед, что групшы автоморфизмов модельных поверхностей, степеней более высоких, чем два, обладают тем же свойством. Это подгрупш групш Кремоны ограниченной степени.

4.5. Вещественные ассоциативные квадрики. Ежов и Шмальц [28] описали очень любопытный класс квадрик типа $(k, k)$. Пусть $\mathbf{A}$ - вешественная $k$-мерная коммутативная ассоциативная алгебра, $\mathbf{A}_{\mathbb{C}}=\mathbf{A} \otimes \mathbb{C}$ - ее комплексификация. Тогда этой алгебре можно сопоставить квадрику

$$
Q=\left\{(z, w) \in \mathbf{A}_{\mathbb{C}} \otimes \mathbf{A}_{\mathbb{C}}: \frac{1}{2 i}(w-\bar{w})=z \bar{z}\right\},
$$

т.е. $\mathbb{R}^{k}$-значная эрмитова форма - это произведение $z \bar{z}$ в алгебре $\mathbf{A}_{\mathbb{C}}$. Обратно, квадрика типа $(k, k)$ допускает такое представление тогда и только тог да, ког да в подходящих координатах форма $\langle\cdot, \cdot\rangle$ определяет вещественное ассоциативное произведение в $\mathbb{R}^{k}$. Коммутативность этого произведения следует из эрмитовости.

Эти квадрики авторы назьвают RAQ-квадриками (Real Associative Quadrics), поэтому мы их будем называть ВАК-квадриками (Вешественные Ассоциативные Квадрики). 
Условие ассощиативности эквивалентно тому, что соответствие

$$
\tau: \mathbf{A} \rightarrow \mathbf{g l}(\mathbb{R}, k), \quad x \mapsto\langle\cdot, x\rangle
$$

является гомоморфизмом алгебр. Невырожденность ВАК-квадрики эквивалентна тому, что А обладает единицей. Распадение квадрики в прямое произведение соответствует разложению алгебры в прямую сумму.

Единственная невырожденная $(1,1)$-квадрика является ВАК-квадрикой. Алгебра, ей соответствуюшая, это $\mathbb{R}$.

Все три невырожденные $(2,2)$-квадрики $Q_{1}, Q_{0}$ и $Q_{-1}$ также являются ВАК-квадриками. Им соответствуют следуюшие алгебры: $\mathbb{R} \otimes \mathbb{R}, \mathbb{C}, \mathbb{R} \otimes \mathbf{n} \mathbb{R}(\mathbf{n}-$ это нильпотентный элемент, $\left.\mathbf{n}^{2}=0\right)$.

$\mathrm{C}(3,3)$-квадриками из списка Палинчак ситуация следуюшая. Квадрики $Q_{1}, Q_{2}$, $Q_{3}$ приводимы (эквивалентны прямому произведению) и, поэтому, являются ВАКквадриками. Из пяти неприводимых квадрик ВАК-квадриками являются лишш две: $Q_{4}$ и $Q_{6}$. Им соответствуют $\mathbb{R} \otimes \mathbf{n}_{1} \mathbb{R} \otimes \mathbf{n}_{2} \mathbb{R}$, где $\mathbf{n}_{1}^{2}=\mathbf{n}_{2}^{2}=\mathbf{n}_{1} \mathbf{n}_{2}=0$ и $\mathbb{R} \otimes \mathbf{n} \mathbb{R} \otimes \mathbf{n}^{2} \mathbb{R}$, где $\mathbf{n}^{3}=0$.

Группа нелинейных автоморфизмов Aut $Q$ BAK-квадрики $Q$ определяется формулой, полностью аналогичной формуле для автоморфизмов $(1,1)$-квадрики:

$$
\begin{gathered}
z \mapsto\left(\operatorname{Id}_{k}-2 i \bar{a} z-(r+i a \bar{a}) w\right)^{-1}(z+a w), \\
w \mapsto\left(\operatorname{Id}_{k}-2 i \bar{a} z-(r+i a \bar{a}) w\right)^{-1} w,
\end{gathered}
$$

где $a \in \mathbf{A}_{\mathbb{C}}$ и $r \in \mathbf{A}$. Таким образом, для ВАК-квадрики $\operatorname{dim} \mathrm{Aut}_{+} Q=3 k$, при этом $\operatorname{dim} g_{1}=2 k$ и $\operatorname{dim} g_{2}=k$. Это, в частности, означает, что в списке Палинчак присутствуют все $(3,3)$-ВАК-квадрики. Причина появления нелинейных автоморфизмов у оставшихся трех квадрик не ясна. Даже среди двух квадрик с самыми богатыми группами $-Q_{5}$ и $Q_{6}$ - одна $\left(Q_{5}\right)$ ВАК-квадрикой не является. Возможно, за этими квадриками скрываются какие-то другие алгебраические структуры.

4.6. Матричная арифметика Ежова-Шмальца. Результаты этого раздела также получены Ежовым и Шмальцом [26]-[29], [31].

Как это видно из классификации Шевченко, $(n, 2)$-квадрики, обладающие нелинейньми автоморфизмами, по аналогии с классификацией $(2,2)$-квадрик можно поделить на гиперболические, эллиптические, параболические и нуль-квадрики. Гиперболические квадрики - это прямое произведение двух гиперквадрик, их можно задать соотношениями:

$$
v_{1}=\sum_{1}^{r} \varepsilon_{i}\left|z_{i}\right|^{2}, \quad v_{2}=\sum_{r+1}^{n} \varepsilon_{i}\left|z_{i}\right|^{2}
$$

эллиптические квадрики имеются только в четной размерности, они задаются соотношениями:

$$
v_{1}=\sum_{1}^{n / 2} \operatorname{Re}\left(z_{2 i-1} \bar{z}_{2 i}\right), \quad v_{2}=\sum_{1}^{n / 2} \operatorname{Im}\left(z_{2 i-1} \bar{z}_{2 i}\right)
$$


параболические:

$$
v_{1}=\sum_{1}^{s} \varepsilon_{i}\left|z_{2 i}\right|^{2}, \quad v_{2}=\sum_{1}^{s} 2 \operatorname{Re}\left(z_{2 i-1} \bar{z}_{2 i}\right)+\sum_{2 s+1}^{n} \varepsilon_{i}\left|z_{i}\right|^{2}
$$

где $\varepsilon_{i}= \pm 1$.

Будем представлять переменное $z$ матрицей $Z$ и переменное $w$ матрицей $W$. Это соответствие определим для каждого типа особо. В гиперболическом случае полагаем:

$$
\begin{aligned}
& Z=\left(\begin{array}{cc}
z_{1} & 0 \\
\vdots & \vdots \\
z_{r} & 0 \\
0 & z_{r+1} \\
\vdots & \vdots \\
0 & z_{n}
\end{array}\right) \\
& \bar{Z}=\left(\begin{array}{cccccc}
\varepsilon_{1} \bar{z}_{1} & \ldots & \varepsilon_{r} \bar{z}_{r} & 0 & \ldots & 0 \\
0 & \ldots & 0 & \varepsilon_{r+1} \bar{z}_{z+1} & \ldots & \varepsilon_{n} \bar{z}_{n}
\end{array}\right), \\
& W=\left(\begin{array}{cc}
w_{1} & 0 \\
0 & w_{2}
\end{array}\right), \quad \bar{W}=\left(\begin{array}{cc}
\bar{w}_{1} & 0 \\
0 & \bar{w}_{2}
\end{array}\right) \text {. }
\end{aligned}
$$

Для эллиптических квадрик

$$
\begin{aligned}
& Z=\left(\begin{array}{cc}
z_{1} & -z_{2} \\
z_{2} & z_{1} \\
\vdots & \vdots \\
z_{n / 2-1} & -z_{n / 2} \\
z_{n / 2} & z_{n / 2-1}
\end{array}\right) \\
& \bar{Z}=\left(\begin{array}{ccccc}
\bar{z}_{1} & -\bar{z}_{2} & \ldots & \bar{z}_{n / 2-1} & -\bar{z}_{n / 2} \\
\bar{z}_{2} & \bar{z}_{1} & \ldots & \bar{z}_{n / 2} & \bar{z}_{n / 2-1}
\end{array}\right), \\
& W=\left(\begin{array}{cc}
w_{1} & -w_{2} \\
w_{2} & w_{1}
\end{array}\right), \quad \bar{W}=\left(\begin{array}{cc}
\bar{w}_{1} & -\bar{w}_{2} \\
\bar{w}_{2} & \bar{w}_{1}
\end{array}\right)
\end{aligned}
$$

и для параболических

$$
\begin{aligned}
& Z=\left(\begin{array}{cc}
z_{1} & 0 \\
z_{2} & z_{1} \\
\vdots & \vdots \\
z_{2 s-1} & 0 \\
z_{2 s} & z_{2 s-1} \\
z_{2 s+1} & 0 \\
z_{n} & 0
\end{array}\right) \\
& \bar{Z}=\left(\begin{array}{cccccccc}
\varepsilon_{1} \bar{z}_{1} & 0 & \ldots & \varepsilon_{s} \bar{z}_{2 s-1} & 0 & 0 & \ldots & 0 \\
\bar{z}_{2} & \bar{z}_{1} & \ldots & \bar{z}_{2 s} & \bar{z}_{2 s-1} & \varepsilon_{2 s+1} \bar{z}_{2 s+1} & \ldots & \varepsilon_{n} \bar{z}_{n}
\end{array}\right) \\
& W=\left(\begin{array}{cc}
w_{1} & 0 \\
w_{2} & w_{1}
\end{array}\right), \quad \bar{W}=\left(\begin{array}{cc}
\bar{w}_{1} & 0 \\
\bar{w}_{2} & \bar{w}_{1}
\end{array}\right) .
\end{aligned}
$$


Во всех трех случаях квадрика $Q$ задается одним и тем же матричным уравнением

$$
\frac{1}{2 i}(W-\bar{W})=\bar{Z} Z
$$

a ее автоморфизмы из Aut $+Q$ задаются единой формулой, вполне аналогичной формуле для дробно-линейных автоморфизмов $(1,1)$-квадрики:

$$
\begin{gathered}
Z \mapsto(Z+A W)(\operatorname{Id}-2 i \bar{A} Z-(R+i A \bar{A}) W)^{-1}, \\
W \mapsto W(\operatorname{Id}-2 i \bar{A} Z-(R+i A \bar{A}) W)^{-1} .
\end{gathered}
$$

Параметры $a \in \mathbb{C}^{n}$ и $r \in \mathbb{R}^{2}$ представлены матрицами $A$ и $R$ в соответствии с теми же правилами, что $z$ и $w$. При этом для всех трех классов $\operatorname{dim} \mathrm{Aut}_{+} Q=2 n+k$, причем $\operatorname{dim} g_{1}=2 n, \operatorname{dim} g_{2}=2$. Формула представляет собой матрично дробно-линейное выражение, поэтому степень автоморфизмов, заданных этой формулой, равна двум, что подтверждает гипотезу о степени (степень не превосходит коразмерности).

Этими тремя классами не исчерпьваются все квадрики коразмерности два с нелинейными автоморфизмами. Последний, четвертый класс, который отсутствует в ситуации $(2,2)$, это так назьваемые нуль-квадрики. Это невырожденные квадрики, заданные такой парой матрищ $H_{1}$ и $H_{2}$, что любая их линейная комбинация вырождена, т.е. их характеристический многочлен $P(t)=\operatorname{det}\left(t_{1} H_{1}+t_{2} H_{2}\right)$ равен нулю тождественно. В каноническом виде это означает присутствие клеток третьего типа. Автоморфизмы нуль-квадрик - это проективные преобразования $\mathbb{C P}^{n+k}$.

Эти построения нашли свое завершение в формуле, дающей общий вид матрично дробно-линейных автоморфизмов произвольной квадрики.

Пусть $\mathbf{A}$ - матричная алгебра вида

$$
\mathbf{A}=\left\{(D, d) \in g l(\mathbb{C}, n) \times g l(\mathbb{C}, k):\langle D z, \bar{z}\rangle=d\langle z, \bar{z}\rangle \text { для всех } z \in \mathbb{C}^{n}\right\} .
$$

Рассмотрим набор полилинейных отображений:

$$
\begin{gathered}
\widehat{A}: \mathbb{C}^{n} \otimes \mathbb{C}^{n} \rightarrow \mathbb{C}^{n} \text { не обязательно симметричньй, } \\
a: \mathbb{C}^{k} \rightarrow \mathbb{C}^{n}, \\
B: \mathbb{C}^{n} \otimes \mathbb{C}^{k} \rightarrow \mathbb{C}^{n}, \\
\widehat{r}: \mathbb{C}^{k} \otimes \mathbb{C}^{k} \rightarrow \mathbb{C}^{k} \text { эрмитов, }
\end{gathered}
$$

которые удовлетворяют соотношениям:

$$
\begin{aligned}
& \langle\widehat{A}(z, \zeta), \xi\rangle=2 i\langle z, \bar{a}\langle\xi, \zeta\rangle\rangle \\
& \langle B(z, w), \zeta\rangle=\widehat{r}(w,\langle\zeta, z\rangle)
\end{aligned}
$$

Тогда набор $(A, a, B, r)$, где $A(z, z)=\widehat{A}(z, z), r(u, u)=\widehat{r}(u, u)$ удовлетворяет соотношениям, определяющим элемент $\mathrm{Aut}_{+} Q$ (см. п. 4.1). Обратное, вообще говоря, не верно. 
Соотношения, определяюшие набор $(\widehat{A}, a, B, \widehat{r})$, эквивалентны тому, что для всех $z$ и $w$

$$
\begin{gathered}
(A(z, \cdot), 2 i\langle z, \bar{a} \cdot\rangle) \in \mathbf{A}, \\
(B(\cdot, w), r(w, \cdot)) \in \mathbf{A} .
\end{gathered}
$$

Пользуясь этим, можно показать, что отображение

$$
\begin{gathered}
z \mapsto\left(\operatorname{Id}_{n}-A(z, \cdot)-B(\cdot, w)-\frac{1}{2} A(a w, \cdot)\right)^{-1}(z+a w), \\
w \mapsto\left(\operatorname{Id}_{k}-2 i\langle z, \bar{a} \cdot\rangle-r(w, \cdot)-i\langle a w, \bar{a} \cdot\rangle\right)^{-1} w
\end{gathered}
$$

является автоморфизмом $Q$. Из-за аналогии между этой формулой и автоморфизмами трехмерной сферы в $\mathbb{C}^{2}$, известными еще Пуанкаре, полученную формулу авторы назвали формулой Пуанкаре. Автоморфизмы этого типа образуют подгруппу групшы автоморфизмов квадрики. Автоморфизмы нуль-квадрики коразмерности два являются дробно-линейными и, поэтому, задаются формулой этого типа. Итак, все автоморфизмы невырожденных квадрик коразмерности два описываются формулой Пуaнкаре.

Первые нелинейные автоморфизмы, не описьваемые этой формулой, были обнаружены в ситуации $(3,3)$. Среди восьми квадрик с нелинейными автоморфизмами, найденных Палинчак, как уже было отмечено, пять $\left(Q_{1}, Q_{2}, Q_{3}, Q_{4}, Q_{6}\right)$ являются ВАК-квадриками и их автоморфизмы, тем самым, задаются формулой Пуанкаре. Формулой Пуанкаре описьваются также автоморфизмы $Q_{5}$ и $Q_{8}$. Квадрика $Q_{7}$ обладает 9 -мерной групой Aut $+Q_{7}$, тогда как подгруппа автоморфизмов Пуанкаре у нее тривиальна.

Когда это было обнаружено, Ежов и Шмальц построили формулу более общего вида, которая описьвает автоморфизмы любой невырожденной квадрики. Мы не приводим здесь этого весьма сложного выражения. Отметим лишш то, что оно является матрично-дробно-квадратичным, т.е. кроме линейных операций и взятия обратного разрешаются произведения двух матричных выражений [31].

\section{5. Квадратичные инварианты $C R$-многообразий: три флаговых конструкции}

Отсутствие у квадрики общего положения нетривиальных автоморфизмов, сохраняющих точку, неэквивалентность двух случайно выбранных квадрик - все это можно рассматривать как проявление одного феномена: наличия у квадрик богатой системы инвариантов относительно действия групшы $G L(n, \mathbb{C}) \oplus G L(k, \mathbb{R})$. Эти же инварианты являются голоморфньми инвариантами дважды гладкой поверхности в комплексном пространстве или даже абстрактной, не обязательно интегрируемой, $C R$-структуры. Первая инвариантная конструкция (характеристический многочлен) появилась в работе Р. Мизнера [41], посвяшенной изучению $C R$-многообразий коразмерности два. Тема $C R$-инвариантов была развита в серии заметок [13]-[16]. В работе Т. Гэррити и Р. Мизнера [33] была описана система образуюших рациональных инвариантных функций. Содержание данного раздела соответствует подходу автора [13]-[16]. 
Первая серия инвариантных множеств $Г(p)$ представляет собой систему вложенных подмножеств пространства $\mathbb{C}^{n}$, заданных однородными полиномиальными условиями. Такие множества естественно рассматривать как алгебраические подмногообразия $\mathbb{C P}^{n-1}$. Пусть $\left(H_{1}, \ldots, H_{k}\right)$ - набор эрмитовых матриц, задающих $(n, k)$-квадрику. Тогда

$$
\Gamma(p)=\left\{z \in \mathbb{C P}^{n-1}: \operatorname{rank}_{\mathbb{C}}\left(H_{1} z, \ldots, H_{k} z\right) \leqslant p\right\} .
$$

То есть множество $\Gamma(p)$ состоит из тех ненулевых векторов $z$, для которых система из $k$ векторов $\left(H_{1} z, \ldots, H_{k} z\right)$ имеет размерность, не превосходящую $p$. При этом

$$
\varnothing=\Gamma(0) \subseteq \Gamma(1) \subseteq \cdots \subseteq \Gamma(k-1) \subseteq \Gamma(k)=\mathbb{C P}^{n-1},
$$

т.е. Г - это система вложенных алгебраических многообразий (флаговая конструкция), занумерованных целыми числами $0 \leqslant p \leqslant k$.

Вторая серия множеств $\gamma(q), 0 \leqslant q \leqslant n$, определяется так:

$$
\gamma(q)=\left\{w \in \mathbb{C P}^{k-1}: \operatorname{rank}\left(w_{1} H_{1}+\cdots+w_{k} H_{k}\right) \leqslant q\right\} .
$$

То есть множество $\gamma(q)$ состоит из тех ненулевых векторов $w$, для которых матрица $\left(w_{1} H_{1}+\cdots+w_{k} H_{k}\right)$ имеет ранг, не превосходящий $q$. Например, $\gamma(n-1)$ - это нулевое множество характеристического многочлена пучка $\left\{P(w)=\operatorname{det}\left(w_{1} H_{1}+\cdots+\right.\right.$ $\left.\left.w_{k} H_{k}\right)=0\right\}$. При этом опять возникает флаговая конструкция из алгебраических подмногообразий проективного пространства

$$
\varnothing=\gamma(0) \subseteq \gamma(1) \subseteq \cdots \subseteq \gamma(n-1) \subseteq \gamma(n)=\mathbb{C P}^{k-1}
$$

Если $n=2$, то $\gamma(n-1) \in \mathbb{C P}^{1}$ это множество собственных значений пучка $w_{1} H_{1}+w_{2} H_{2}$, а $\Gamma(2) \in \mathbb{C P}^{n-1}$ это множество собственных векторов, отвечающих собственным значениям из $\gamma(n-1)$. Поэтому в общем случае полученные флаговые конструкции являются естественным обобшением этих задач: серия $\gamma$ - задачи на собственные значения, а серия $\Gamma$ - на собственные векторы.

Третья флаговая конструкция $\delta$ возникает при рассмотрении особенностей проективизации квадрики. Если от аффинных координат $(z, w), z \in \mathbb{C}^{n}, w \in \mathbb{C}^{k}$, перейти к проективньм координатам $(T, Z, W), T \in \mathbb{C}^{1}, Z \in \mathbb{C}^{n}, W \in \mathbb{C}^{k}$, с помошью замен $z=Z / T, w=W / T$, то уравнение $\bar{Q}$ замькания квадрики $Q$ будет иметь вид

$$
\operatorname{Im} W \bar{T}=\langle Z, \bar{Z}\rangle .
$$

Проективизация гиперквадрики является проективно однородной, проективные автоморфизмы действуют на ней транзитивно, гиперквадрика не имеет особенностей на бесконечности. Однако при $k \geqslant 2$ это не так. Гиперповерхности, пересечение которых образует квадрику, могут пересекаться на бесконечности $\{T=0\}$ не трансверсально. Самая грубая характеристика возникаюших здесь особенностей это размерность касательного пространства $T(\bar{Q})$, которая дает стратификацию точек $Q^{\infty}=\bar{Q} \backslash Q$. Эта размерность определяется рангом системы градиентов, определяюших квадрику функций. Пусть $e_{j}(T, Z, W)=\operatorname{grad}\left(-\operatorname{Im} W_{j} \bar{T}+\langle Z, \bar{Z}\rangle\right)$, тогда

$$
\delta(r)=\left\{(T, Z, W) \in \mathbb{C P}^{n+k}: \operatorname{rank}_{\mathbb{C}}\left(e_{1}(T, Z, W), \ldots, e_{k}(T, Z, W)\right) \leqslant r\right\} .
$$


Система $\delta$ также является фллаговой конструкцией алгебраических подмножеств

$$
\varnothing=\delta(0) \subseteq \delta(1) \subseteq \cdots \subseteq \delta(n-1) \subseteq \delta(k)=\mathbb{C P}^{n+k}
$$

Все три серии определялись неравенствами на ранги в комплексном пространстве. Можно получить более тонкую стратификацию $Q^{\infty}$, рассмотрев вешественные аналоги этих флаговых конструкций:

$$
\begin{gathered}
\Gamma R(p)=\left\{z \in \mathbb{R}^{2 n-1}: \operatorname{rank}_{\mathbb{R}}\left(H_{1} z, \ldots, H_{k} z\right) \leqslant p\right\}, \\
\gamma R(q)=\left\{u \in \mathbb{R}^{k-1}: \operatorname{rank}\left(u_{1} H_{1}+\cdots+u_{k} H_{k}\right) \leqslant q\right\}, \\
\delta R(r)=\left\{(T, Z, W) \in \mathbb{R}^{2 n+2 k}: \operatorname{rank}_{\mathbb{R}}\left(e_{1}(T, Z, W), \ldots, e_{k}(T, Z, W)\right) \leqslant r\right\} .
\end{gathered}
$$

Отметим также еще два инвариантных множества: эрмитов световой конус $S=$ $\left\{z \in \mathbb{R P}^{2 n-1}:\langle z, \bar{z}\rangle=0\right\}$ и нуль билинейной формы $S^{\prime}=\left\{(z, \zeta) \in \mathbb{C P}^{2 n-1}:\right.$ $\langle z, \zeta\rangle=0\}$.

Если имеются два ростка, эквивалентные посредством голоморфного или $C R$-отображения, то их касательные квадрики эквивалентны, причем эквивалентность устанавливает линейное отображение вида $z \rightarrow C z, w \rightarrow \rho w$. Это и есть причина того, что вся совокупность перечисленных множеств первой квадрики оказьвается линейно эквивалентной соответствующим множествам второй. Причем эквивалентность устанавливается:

для флагов $\Gamma$ и $\Gamma R$, а также $S_{1}, S_{2}, S_{3}$ посредством индуцированного действия $C$ на $\mathbb{C P}^{n-1}$ и $\mathbb{R P}^{2 n-1}$,

для флагов $\gamma$ и $\gamma R$ посредством индуцированного действия $\rho^{\prime}$ - матрицы, полученной транспонированием из $\rho$ на $\mathbb{C P}^{k-1}$ и $\mathbb{R} \mathbb{P}^{k-1}$,

для флагов $\delta$ и $\delta R$ посредством индуцированного действия пары $(C, \rho)$ на $\mathbb{C P}^{n+k}$ и $\mathbb{R} \mathbb{P}^{2 n+2 k}$

То, что на переменную $w$ действуют вешественные замены, означает, что вешественное подпространство $\mathbb{R}^{k}=\{\operatorname{Im} w=0\}$ остается инвариантным и $\gamma R$ - это не что иное, как $\gamma \cap \mathbb{R}^{k}$.

Характеристический многочлен $P(w)=\operatorname{det}\left(w_{1} H_{1}+\cdots+w_{k} H_{k}\right)$ преобразуется следуюшим образом: $P(w) \rightarrow|\operatorname{det} \Lambda|^{-2} P\left(\rho^{\prime} w\right)$.

Таким образом, все линейные инварианты всех перечисленных множеств, построенных по некоторой форме $\langle z, \bar{z}\rangle$, являются биголоморфными инвариантами квадрики и ростка поверхности, а также $C R$-инвариантами ростка $C R$-многообразия с данной формой Леви. Среди этих инвариантов, в первую очередь, следует отметить наборы размерностей и степеней. В этот список целочисленных инвариантов следует также включить набор размерностей градуированных компонент $\left(\operatorname{dim} g_{0}, \operatorname{dim} g_{1}, \operatorname{dim} g_{2}\right)$. Биголоморфная (или $C R$ ) эквивалентность двух вещественных многообразий означает линейную эквивалентность всех построенных множеств и равенство всех их линейных инвариантов в каждой паре соответствуюших точек. 
Для $(2,2)$-квадрик все флаги $\Gamma, \Gamma R, \gamma, \gamma R, \delta, \delta R$ имеют лишш одну содержательную позицию - первую. Для трех стандартных $(2,2)$-квадрик картина следующая:

$$
\begin{array}{rll}
Q_{1}: & \Gamma(1)-\text { две точки; } & \Gamma R(1)-\text { две точки; } \\
& \gamma(1)-\text { две точки; } & \gamma R(1)-\text { две точки; } \\
& \delta(1)-\text { одна точка; } & \delta R(1)-\text { одна точка; } \\
Q_{0}: & \Gamma(1)-\text { одна точка; } & \Gamma R(1)-\text { две прямых; } \\
& \gamma(1)-\text { одна точка; } & \gamma R(1)-\text { одна точка; } \\
& \delta(1)-\text { две точки; } & \delta R(1)-\text { одна точка и одна прямая; } \\
Q_{-1}: & \Gamma(1)-\text { две точки; } & \Gamma R(1)-\varnothing ; \\
& \gamma(1)-\text { две точки; } & \gamma R(1)-\varnothing ; \\
\delta(1)-\text { три точки; } & \delta R(1)-\text { одна точка. }
\end{array}
$$

Для различения $(3,3)$ и $(3,4)$-квадрик из списков Палинчак и Анисовой достаточно характеристического многочлена $P(w)$ и светового конуса $S$. Начнем со списка Палинчак.

$$
\begin{aligned}
& Q_{1}: \quad P(w)=w_{1} w_{2} w_{3}, \quad \gamma R(2)-\text { три прямые; } \\
& Q_{2}: \quad P(w)=-w_{3}\left(w_{1}+i w_{2}\right)\left(w_{1}-i w_{2}\right), \quad \gamma R(2) \text { - одна прямая; } \\
& Q_{3}: \quad P(w)=-\left(w_{2}\right)^{2} w_{3}, \quad \gamma R(2)-\text { две прямые; } \\
& Q_{4}: \quad P(w)=-\left(w_{3}\right)^{3}, \quad \gamma R(2)-\text { одна прямая, } \\
& S \text { - одна прямая; } \\
& Q_{5}: \quad P(w)=0, \quad \gamma R(2)-\text { все пространство, } \\
& S \text { - конус степени четыре; } \\
& Q_{6}: \quad P(w)=0, \quad \gamma R(2)-\text { все пространство, } \\
& S \text { - трехмерное подпространство; } \\
& Q_{7}: \quad P(w)=0, \quad \gamma R(2)-\text { все пространство, } \\
& S \text { - трехмерное подпространство и квадратичньй конус; } \\
& Q_{8}: \quad P(w)=-\left(w_{3}\right)^{3}, \quad \gamma R(2) \text { - одна прямая, } \\
& S-\text { квадратичньй конус. }
\end{aligned}
$$

Этого достаточно, чтобы убедиться в попарной неэквивалентности этих квадрик. Если для доказательства неэквивалентности привлечь размерности компонент алгебры, то в списке Анисовой отличить остается лишь $Q_{3}$ от $Q_{4}$ и $Q_{5}$ от $Q_{9}$. Первая пара различается строением конуса $S$. У $Q_{3}$ он плоский, а у $Q_{4}-$ нет. Вторая - характеристическим многочленом. У $Q_{3}$ разложение на неприводимые компоненты содержит 
кратный линейньй множитель $\left(P(w)=w_{1}^{2}\left(w_{1}+w_{2}\right)\right)$, а у $Q_{4}$ все три линейных множителя различны $\left(P(w)=w_{4}\left(w_{1}+i w_{2}\right)\left(w_{1}-i w_{2}\right)\right)$.

Для $(3,3)$-квадрики общего положения как $Г(2)$, так и $\gamma(2)$ являются неособыми кубическими кривыми в $\mathbb{C P}^{2}$. Как известно, такая кривая имеет 9 точек перегиба, причем эта девятка точек не обладает проективными симметриями (двойные отношения всех четверок попарно различны). Пользуясь этой асимметрией, точки можно упорядочить, так что если имеется проективное отображение одной кубики на другую, то оно переведет точки перегиба первой кубики в точки перегиба второй с сохранением порядка (первую в первую, и т. д.). При отображении квадрики на себя действие на комплексной касательной сводится, таким образом, к скалярным растяжениям $\{z \rightarrow \lambda z\}$, где $\lambda$ - ненулевой комплексный множитель. Это и является геометрической причиной жесткости квадрики.

Для многообразий с жесткими касательньми квадриками вопросы классификации решаются весьма просто [13], [16]. В частности, приведение локального уравнения такого многообразия к некоторой нормальной форме позволяет вьписать полную систему локальных инвариантов ростка.

Описанные инвариантные конструкции не только позволяют получать запреты на эквивалентность ростков, но и формулировать запреты на существование необратимых голоморфных отображений одного ростка в другой. Конечно, всегда есть возможность постоянного отображения в любой росток, поэтому такие запреты имеют характер оценок на степень вырождения (ранг касательного отображения). Причем это касается не только отображений квадрик, но и отображений ростков гладких многообразий разных типов [15]. Запреты для квадрик, конечно, означают запреты и для ростков с данными касательными квадриками.

Приводимые ниже утверждения делаются для квадрик обшего положения на основе данных о размерностях инвариантных множеств, однако так же можно исследовать и особые квадрики. Итак, для всех перечисленных ниже пар можно утверждать, что если имеется отображение первой квадрики во вторую, то ранг его касательного отображения меньше максимально возможного по крайней мере на две единицы. Вот список пар:

1. гиперквадрика типа $(3,1)$ в $(3,3)$-квадрику;

2. гиперквадрика типа $(4,1)$ в $(4,3)$-квадрику;

3. (3,2)-квадрика в $(3,3)$-квадрику;

4. (3, 3)-квадрика в $(4,4)$-квадрику;

5. (3, 3)-квадрика в $(3,5)$-квадрику.

\section{6. Модели высших порядков}

В предыдуших разделах мы попытались продемонстрировать, что невырожденная вещественная квадрика в комплексном пространстве является объектом, замечательным во многих отношениях, и что описание автоморфизмов, построение системы инвариантов и классификация для ростков поверхностей (ростков $C R$-многообразий) могут опираться на решение этих задач для касательной квадрики. То, что такой объект сушествует, является большой удачей. Это факт, достойньй удивления. Покажем 
это на проблеме выбора степени модели (это та степень $d$, которая для квадрики равна двум). Отдельно взятые требование универсальности и однородности оба "работают" на снижение $d$. Действительно, модель степени один вещественного многообразия $\{\operatorname{Im} w=0\}$ и универсальна, и однородна. Требование конечномерности (групшы автоморфизмов) “работает" на повышение $d$ : росток поверхности степени больше, чем два, общего положения (некая невырожденность) имеет конечномерную группу. То, что эти две противоборствуюшие тенденщии смогли “согласиться" на $d=2$ - это удача. И остальные требования из списка требований к хорошей модели можно рассматривать как бесплатное приложение.

Однако при росте коразмерности возникает некоторое осложнение. Дело в том, что размерность пространства эрмитовых матриц порядка $n$ конечна и равна $n^{2}$. Если коразмерность превзойдет этот рубеж, то первая часть условия конечномерности становится невыполнимой. Это приводит к тому, что все квадрики такого типа оказываются вырожденными, а их групшы бесконечномерньми. Таким образом, при $k>n^{2}$ невозможно сформулировать критерий конечномерности группы ростка типа $(n, k)$ в терминах 2-струи, и возникает необходимость в построении новой хорошей модели.

На это явление можно посмотреть с другой стороны. Если $T^{c}(M)$ - расслоение вешественных векторных полей на $M$, принадлежаших в каждой точке комплексной части касательной плоскости, то градуированная алгебра Леви-Танаки, индуктивно определяется соотношениями

$$
D^{1}=T^{c}(M), \quad D^{j+1}=\left[D^{j}, D^{1}\right]+D^{j}
$$

(скобка - это коммутатор полей). Если эта последовательность стабилизируется на $l$-м шаге $D^{l-1} \neq D^{l}=D^{l+1}=\cdots=D^{\infty}$, говорим, что длина алгебры равна $l$. Невырожденность формы Леви означает тогда в точности то, что длина алгебры равна двум, причем $D^{\infty}=T(M)$. Ясно, что при $k>n^{2}$ это невозможно. Высшие коразмерности требуют привлечения коммутаторов более высоких порядков, что в координатных терминах означает переход к рассмотрению струй порядка вьше, чем два.

Есть еще одно обстоятельство, побуждающее перейти к рассмотрению более высоких степеней. Все работы по квадратичным моделям были выполнены во II-м тысячелетии, и наступление III-го тысячелетия побуждает перейти к рассмотрению моделей степени три и выше.

6.1. Кубическая модель. Существует, с точностью до эквивалентности, всего одна невырожденная эрмитова форма типа $\left(n, n^{2}\right)$. Это форма, компонентами которой является произвольный набор из $n^{2}$ скалярных линейно независимых эрмитовых форм. Обозначим эту форму через $\langle z, \bar{z}\rangle$. Поверхностью типа $\left(n, K=n^{2}+k\right)$, $k>0$, которая является полным аналогом невырожденной квадрики, т.е. поверхностью, для которой вьполнен полный список требований, предъявляемых к хорошей модели, является поверхность $Q_{3}$, заданная в пространстве $\mathbb{C}^{n} \oplus \mathbb{C}^{n^{2}} \oplus \mathbb{C}^{k}$ с координатами $\left(z \in \mathbb{C}^{n}, w_{2} \in \mathbb{C}^{n^{2}}, w_{3} \in \mathbb{C}^{k}\right), n>0, k>0$, уравнениями

$$
\operatorname{Im} w_{2}=\langle z, \bar{z}\rangle, \quad \operatorname{Im} w_{3}=2 \operatorname{Re} \Phi(z, z, \bar{z}),
$$

где $\Phi(z, z, \bar{z})$ - однородная форма степени два по $z$ и степени один по $\bar{z}$ со значениями B $\mathbb{C}^{k}$. 
Говорим, что поверхность $Q_{3}$ является касательной кубикой ростка того же типа, если его локальные уравнения можно записать в виде

$$
\operatorname{Im} w_{2}=\langle z, \bar{z}\rangle+F_{3}+\cdots, \quad \operatorname{Im} w_{3}=2 \operatorname{Re} \Phi(z, z, \bar{z})+G_{4}+\cdots
$$

(при подсчете весов пользуемся правилом: $[z]=1,\left[\operatorname{Re} w_{2}\right]=2,\left[\operatorname{Re} w_{3}\right]=3$ ).

ОПРЕДЕЛЕНИЕ. Поверхность (15) называется невьрожденной, если $\langle z, \bar{z}\rangle-$ это невырожденная эрмитова форма типа $\left(n, n^{2}\right)$, а координаты формы $\Phi(z, z, \bar{z})$ линейно независимы.

Подобно тому, как форме Леви можно дать инвариантное определение, такое определение можно дать и кубической форме $\Phi$. Для этого следует рассмотреть повторньй коммутатор $(1,0)$-полей $[17]$. Длина алгебры Леви-Танаки поверхности $Q_{3}$ равна 3 .

Использование таких поверхностей имеет свои ограничения, вытекающие из того, что размерность пространства кубических форм конечна и равна $n^{2}(n+1)$. Итак, то, что невырожденная поверхность $Q_{3}$ типа $\left(n, K=n^{2}+k\right)$ при $k \leqslant n^{2}(n+1)$ является хорошей модельной поверхностью невырожденных ростков того же типа, можно выразить тем же списком свойств [17], который при $d=3$ получает следующую редакцию.

1. Универсальность: любой порождающий Леви-невырожденньй росток многообразия типа $\left(n, K=n^{2}+k\right)$ в $\mathbb{C}^{n+K}$ эквивалентен ростку вида (15);

2. Конечномерность: (а) группа голоморфных автоморфизмов поверхности $Q_{3}$ общего положения является конечномерной группой Ли; (b) критерием конечномерности групшы голоморфных автоморфизмов $Q_{3}$ является невырожденность, т.е. линейная независимость всех координатных форм; (c) любая поверхность данного типа, заданная уравнениями степени меньшей, чем три, имеет бесконечномерную групу;

3. Однородность: $Q_{3}$ однородна, т.е. ее голоморфные автоморфизмы действуют на ней транзитивно, однородность обеспечивается квадратично-треугольными преобразованиями;

4. Симметричность: (а) кубика является самой симметричной невырожденной поверхностью, т.е. размерность группы ростка невырожденной поверхности не превосходит размерности группы ее касательной кубики; (b) алгебра автоморфизмов кубики параметризует семейство отображений одного невырожденного ростка в другой;

5. Алгебраические свойства: (а) алгебра Ли голоморфных векторных полей на невырожденной кубике - это некоторая алгебра полиномиальных векторных полей ограниченной степени, степени коэффициентов не превосходят пяти; (b) группа автоморфизмов невырожденной кубики - это група Ли, являюшаяся подгруппой группы бирациональных преобразований $\mathbb{C}^{n+K}$ ограниченной степени, в качестве оценки степени числителей и знаменателей в несократимом представлении можно взять $15(n+K) ;(c)$ два ростка эквивалентны лишь в том случае, если эквивалентны их касательные кубики, две кубики голоморфно эквивалентны в том и только том случае, когда они эквивалентны линейно. 
Относительно строения алгебры автоморфизмов кубики Aut $Q_{3}$ можно сказать следуюшее. Введем градуировку в пространстве векторных полей с коэффициентами, зависящими от $\left(z, w_{2}, w_{3}\right)$, полагая

$$
[z]=1, \quad\left[w_{2}\right]=2, \quad\left[w_{3}\right]=3\left[\frac{\partial}{\partial z}\right]=-1, \quad\left[\frac{\partial}{\partial w_{2}}\right]=-2, \quad\left[\frac{\partial}{\partial w_{3}}\right]=-3
$$

тогда алгебра Aut $Q_{3}$ становится градуированной алгеброй Ли вида

$$
g_{-3}+g_{-2}+g_{-1}+g_{0}+g_{1}+g_{2}+g_{3}+g_{4}+g_{5}+g_{6}
$$

При этом подалгебра $g_{-}=g_{-3}+g_{-2}+g_{-1}-$ это алгебра Ли группы треугольно-квадратичных преобразований Aut_ $Q_{3}$ вида

$$
\begin{gathered}
z \rightarrow p+z, \\
w_{2} \rightarrow(q+i\langle p, \bar{p}\rangle)+2 i\langle z, \bar{p}\rangle+w_{2}, \\
w_{3} \rightarrow(r+2 i \operatorname{Re} \Phi(p, p, \bar{p}))+4 i \Phi(z, p, \bar{p})+2 i \Phi(z, z, \bar{p})+2 i \bar{\Phi}(\bar{p}, \bar{p}, z)+s w_{2}+w_{3} ;
\end{gathered}
$$

причем $(p, q, r)$ произвольная точка $Q_{3}$ и

$$
\operatorname{Re} \Phi(z, p, \bar{z})=s\langle z, \bar{z}\rangle \text {. }
$$

Tо есть Aut_ $Q_{3}$ - это та подгруппа, которая обеспечивает однородность $Q_{3}$, соответственно $\operatorname{dim}$ Aut $-Q_{3}=\operatorname{dim} Q_{3}=2 n+n^{2}+k$.

Подалгебра $g_{0}$ соответствует линейньм автоморфизмам кубики

$$
z \rightarrow \Lambda z, \quad \zeta \rightarrow \rho \zeta, \quad w \rightarrow \nu w
$$

где

$$
\langle\Lambda z, \overline{\Lambda z}\rangle=\rho\langle z, \bar{z}\rangle, \quad \Phi(\Lambda z, \Lambda z, \overline{\Lambda z})=\nu \Phi(z, z, \bar{z}) .
$$

Размерность этой группы может меняться в зависимости от кубической формы $\Phi$. Но, как видно из определяюших соотношений, если фиксировать значение невырожденной матрицы $\Lambda$, то значения $\rho$ и $\nu$ определяются однозначно. Поэтому справедлива оценка $\operatorname{dim} \mathrm{Aut}_{0} Q \leqslant 2 n^{2}$. Эту подг руппу автоморфизмов кубики обозначим $\mathrm{Aut}_{0} Q$.

Действие голоморфных замен на ростки порождает линейное действие на касательные кубики групшы $G L(n, \mathbb{C}) \oplus G L(k, \mathbb{R})$ вида

$$
\Phi(z, z, \bar{z}) \rightarrow \nu \Phi\left(\Lambda^{-1} z, \Lambda^{-1} z, \overline{\Lambda^{-1} z}\right) .
$$

Подалгебра $g_{+}=g_{1}+g_{2}+g_{3}+g_{4}+g_{5}+g_{6}$ это нильпотентная алгебра Ли. Ей соответствует подгрупша нелинейных автоморфизмов кубики, сохраняющих начало координат. Причем таких, что $\Lambda=E_{n}, \rho=E_{n^{2}}, \nu=E_{k}$. Эту группу мы обозначим через Aut $+Q$. В разобранных примерах: $(1,2)[19],(1,3),(1,4),(1,5),(1,6)[51]$, $\left(n, n^{2}+1\right)[49]$ этой подалгебры просто нет $(=0)$ и все автоморфизмы, сохраняюшие точку, линейные. Таким образом, вопрос о существовании кубики с нетривиальной подгруппой Aut $Q_{3}$ открыт. При этом, по-видимому, как и в случае с квадриками (жесткость), для кубики общего положения таких автоморфизмов нет, и речь идет о поиске исключительных кубик. 
6.2. Квазипериодическая система модельных поверхностей. Приведенная для $d=2,3$ конструкция имеет обобщение для многообразий с произвольно большой длиной алгебры Леви-Танаки [18]. Процесс порождения модельных поверхностей все более высоких степеней напоминает процесс заполнения электронами разрешенных энергетических уровней в атоме [38]. При этом вся серия напоминает периодическую систему элементов Д. И. Менделеева.

Пусть $\mathscr{F} m, n-$ пространство вещественных многочленов от $\left(z_{1}, \ldots, z_{n}, \bar{z}_{1}, \ldots, \bar{z}_{n}\right)$ степени $m$, чье разложение по бистепеням не содержит компонент $(m, 0)$ и $(0, m)$. Тогда если $k_{m, n}=\operatorname{dim} \mathscr{F}_{n, m}$, то $k_{m, n}=C_{2 n+m-1}^{m}-2 C_{n+m-1}^{m}$, если $K_{m, n}=k_{2, n}+$ $k_{3, n}+\cdots+k_{m, n}$, то $K_{m, n}=C_{2 n+m}^{m}-2 C_{n+m}^{m}+1$. В частности,

$$
k_{2, n}=n^{2}, \quad k_{3, n}=n^{2}(n+1), \quad k_{4, n}=n^{2}(n+1)(7 n+1) / 12 .
$$

Модельная поверхность $Q_{d}$ типа $(n, K)$ степени $d$, где $K_{d-1, n}<K \leqslant K_{d, n}$, это поверхность, заданная в пространстве

$$
\mathbb{C}^{n} \oplus \mathbb{C}^{k_{2, n}} \oplus \cdots \oplus \mathbb{C}^{k_{d-1, n}} \oplus \mathbb{C}^{k},
$$

$k=K-K_{d-1, n}$, с координатами $\left(z, w_{2}, \ldots, w_{d}\right)$ соотношениями

$$
\operatorname{Im} w_{m}=\Phi_{m}(z, \bar{z}), \quad m=2, \ldots, d,
$$

причем $\Phi_{m}$ для $m=2, \ldots, d-1-$ это вектор, составленньй из базисных элементов пространства $\mathscr{F}_{m}$, а $\Phi_{d}$ - вектор из линейно независимых элементов $\mathscr{F}_{d}$. При $d=2$ мы получаем невырожденную квадрику, а при $d=3$ - невырожденную кубику. Подчеркнем, что степень $d$ не является независимым параметром в отличие от $n$ и $K$, а определяется условием попадания в определенный интервал значений.

Поверхность $Q_{d}$ будем назьвать касательной модельной поверхностью для ростка вида

$$
\begin{aligned}
\operatorname{Im} w_{2} & =\Phi_{2}+\text { члены веса три и более, } \\
\cdots & \\
\operatorname{Im} w_{d} & =\Phi_{d}+\text { члены веса }(d+1) \text { и более. }
\end{aligned}
$$

При подсчете весов принято очевидное соглашение $\left[\operatorname{Re} w_{j}\right]=j$. Вешественно алгебраическую поверхность $Q_{d}$ вида $(15)$ будем назьвать касательной модельной поверхностью ростка $M_{0}$ вида (16). Поверхность и росток будем называть невырожденными, если все координатные формы $\Phi$ линейно независимы.

Поверхности серии $Q_{d}$ удовлетворяют всем требованиям, предъявляемым к хорошим моделям, за одним, но сушественным, исключением. Речь идет о требовании универсальности. Этому требованию $Q_{d}$ удовлетворяет при $d=2$ и 3 . Используя простейшие замены, нетрудно показать, что любой росток типа $(n, K)$, где $K_{3}<K \leqslant K_{4}$, с невырожденной 3-струей можно записать в виде

$$
\begin{aligned}
& \operatorname{Im} w_{2}=\Phi_{2}(z, \bar{z})+\text { члены веса три и более, } \\
& \operatorname{Im} w_{3}=\Phi_{3}(z, \bar{z})+\text { члены веса четыре и более, } \\
& \operatorname{Im} w_{4}=\Phi_{4}(z, \bar{z})+\text { члены веса пять и более, }
\end{aligned}
$$


что доказьвает универсальность данной модели при $d=4$. При $d \geqslant 5$ это уже не так. Причина в том, что доля переменных группы $z$ (комплексная касательная) становится слишком малой в общей совокупности координат пространства. Это приводит к тому, что не удается выбором локальных координат обеспечить правильное касание ростка и нашей модельной поверхности. Выход очевиден: следует расширить класс модельных поверхностей, разрешив некоторую зависимость от переменных групшы Re $w$. Это можно сделать, не теряя однородности. Однако узловой пункт нашей программы - это получение критерия конечномерности. Конечномерность, на уровне алгебры, вместе с полиномиальностью и оценкой степени была получена из анализа линейной системы дифференциальных уравнений. Переход к моделям с зависимостью от $\operatorname{Re} w$ означает, что система перестает быть системой с постоянными коэффициентами. Это приводит к тому, что дифференциальные операторы, с помошью которых записьвается система, перестают коммутировать. В связи с этой некоммутативностью возникают технические препятствия, которые, как можно надеяться, будут в ближайшем будушем преодолены.

С модельными поверхностями высоких степеней связан еще один мало исследованный вопрос. Положительно определенные квадрики являются остовами однородных областей. Каждой модельной поверхности можно сопоставить ее оболочку голоморфности. Это некая область в $\mathbb{C}^{n+K}$. Если модельная поверхность является в каком-либо смысле вьпуклой ( “положительная определенность"), то оболочка является областью, не совпадающей со всем пространством, если поверхность можно поместить в острьй конус, то она оказьвается областью, биголоморфно эквивалентной ограниченной. Однородность остова позволяет надеяться на то, что однородной будет и сама оболочка. Если это так, то модельные поверхности могут оказаться источником новых примеров однородных ограниченных областей (новые области Зигеля).

\section{7. Поверхности с вырождениями}

На протяжении всей данной работы при построении модельных поверхностей мы последовательно уходили от рассмотрения "вырожденных" ситуаций. В этом разделе мы коснемся этой проблематики. Это позволит нам сформулировать, в качестве гипотезы, еше одно свойство хороших модельных поверхностей. В этом разделе все рассматриваемые поверхности будем считать вещественно аналитическими и связными.

Рассмотрим опять ту простейшую ситуацию, с которой мы начинали. Пусть $M-$ связная поверхность типа $(1,1)$, т.е. гиперповерхность в $\mathbb{C}^{2}$, и $\xi$ - это ее точка. Если форма Леви гиперповерхности в точке $\xi$ невырождена, то, как нам хорошо известно, $\operatorname{dim}$ Aut $M_{\xi} \leqslant \operatorname{dim}$ Aut $Q=8$, где $Q$ - единственная модельная поверхность данного типа. Если форма в точке вырождена, то $M$ содержит непустое аналитическое подмножество $M_{0}=\{p \in M: M$ вырождена в $p\}$. Если $M_{0}$ содержит открытые части $M$, то по теореме единственности $M_{0}=M$. В этом случае из теоремы $Ф$ робениуса следует, что в некоторых голоморфных координатах в окрестности любой своей точки гиперповерхность $M$ является гиперплоскостью. В частности, $\operatorname{dim} \mathrm{Aut} M_{\xi}=\infty$ и это можно сказать о любой точке этой гиперповерхности.

Оказьвается, что это единственная возможность для ростка гиперповерхности иметь бесконечномерную группу. Более того, можно утверждать, что если гиперпо- 
верхность не является локально плоской, то в любой ее точке, как в невырожденной, так и в вырожденной, $\operatorname{dim}$ Aut $M_{\xi} \leqslant \operatorname{dim} \operatorname{Aut} Q=8$.

Доказать это несложно. Рассмотрим произвольные девять векторных полей $\left(X_{1}, \ldots, X_{9}\right)$ из aut $M_{\xi}$, определенные в окрестности $U_{\xi}$ точки $\xi$. Коль скоро $M$ не является локально плоской, то $M \backslash M_{0}$ есть открытоеплотноеподмножество $M$, ипусть $\xi^{\prime}$-произвольная точка из $\left(M \backslash M_{0}\right) \cap U_{\xi}$. В точке $\xi^{\prime}$ гиперповерхность $M$ невырождена, поэтому из девяти полей можно составить нетривиальную линейную комбинацию с вешественньми коэффициентами, тождественно равную нулю в окрестности $\xi^{\prime}$. Эти поля имеют голоморфные коэффищиенты в полномерной окрестности $\xi$, их сужения на $M$ имеют вешественно аналитические коэффищиенты, поэтому обрашение в нуль линейной комбинации по теореме единственности продолжается из окрестности точки $\xi^{\prime}$ на всю окрестность $U_{\xi}$. Это завершает доказательство.

Итак, если $M-$ связная вещественно аналитическая гиперповерхность в $\mathbb{C}^{2}$ и $\xi$-ее точка, то имеет место следующая

Альтернатива (для гиперповерхности в $\mathbb{C}^{2}$ ):

либо $\operatorname{dim}$ Aut $M_{\xi}=\infty$ и $M$ в окрестности любой своей точки эквивалентна гиперплоскости;

либо $\operatorname{dim}$ Aut $M_{\xi} \leqslant \operatorname{dim}$ Aut $Q(1,1)=8$.

Таким образом, гиперквадрика является самой симметричной гиперповерхностью не только в классе невырожденных ростков, но и в классе всех ростков с конечномерной группой.

Если рассмотреть вместо всей групшы подгруппу Aut ${ }_{\xi} M_{\xi}$ автоморфизмов, оставляюших точку $\xi$ на месте (подгруппа стабильности), то ее размерность для гиперквадрики в $\mathbb{C}^{2}$ равна пяти. Нам не известно ни одного примера поверхности с конечномерной группой, чья подгрупша стабильности была бы больше. Однако доказательства нет, и вопрос открыт.

Вопрос о возможности локального распрямления поверхности был изучен в работе Фримана [32]. Росток поверхности $M_{\xi}$ в $\mathbb{C}^{N}$ назьвается $q$-распрямляемым, если он биголоморфно эквивалентен ростку вида $\widetilde{M}_{0} \times \mathbb{C}^{q}$, где $\widetilde{M}_{0}-$ росток поверхности в $\mathbb{C}^{N-q}$. Росток распрямляем, если он $q$-распрямляем для некоторого $q>0$. Рассмотрения Фримана основаны на использовании теоремы $Ф$ робениуса.

Критерий конечномерности группы автоморфизмов гиперповерхности предложен Н. Стентон [54]. Его удобнее формулировать как критерий бесконечномерности: $\operatorname{dim}$ aut $M_{\xi}=\infty$ тогда и только тогда, когда не существуют ненулевые касательные $(1,0)$-поля с голоморфными коэффициентами. Критерий не является тривиальной перефформулировкой, однако его проверка - это вьгислительная задача того же типа, что и вычисление алгебры автоморфизмов. Ростки с конечномерной группой Стентон называет голоморфно невырожденньми. На связной вешественно аналитической поверхности голоморфная невырожденность вьполняется (или не выполняется) для всех точек одновременно ([5], см. также [37]).

Приведенное вьше доказательство альтернативы не переносится даже на гиперповерхности в $\mathbb{C}^{3}$. Форма Леви такой гиперповерхности - это эрмитова форма на $\mathbb{C}^{2}$, и ее ранг может принимать три значения: 0,1 и 2. Если ранг равен нулю на открытой части гиперповерхности, то поверхность распрямляемая и размерность групшы равна 
бесконечности (голоморфная вырожденность). Если ранг равен двум, то это означает, что форма невырождена. Если ранг равен двум хотя бы в одной точке, то он равен двум на открытом плотном подмножестве гиперповерхности. Это позволяет, повторяя наше рассуждение, получить оценку $\operatorname{dim}$ Aut $M_{\xi} \leqslant \operatorname{dim} \operatorname{Aut} Q(2,1)=15$. Однако ранг может повсюду быть равным единище. Примером такой гиперповерхности является световой конус:

$$
\left\{\left(z_{1}, z_{2}, w\right) \in \mathbb{C}^{3}:(\operatorname{Im} w)^{2}=\left(\operatorname{Im} z_{1}\right)^{2}+\left(\operatorname{Im} z_{2}\right)^{2}\right\}
$$

Альтернатива остается справедливой, но ее доказательство требует бо́льших усилий.

В работах Баоуэнди, Ротшильд, Эбенфельта, Хуанга и Зайцева [5]-[9] был рассмотрен ряд характеристик вырожденных точек вешественного многообразия. В частности, была рассмотрена такая характеристика точки, как $l$-невырожденность. Многообразие $M$ назьвается конечно невырожденным в точке $\xi$, если существует такое $l \geqslant 0$, что для любого $(0,1)$-векторного поля $L$ на $M$ с $L(\xi) \neq 0$ найдутся такие $(0,1)$-поля $L_{1}, \ldots, L_{m}, 0 \leqslant m \leqslant l$, что $\left[L_{1}, \ldots,\left[L_{m}, \bar{L}\right], \ldots\right](\xi) \notin T_{\xi}^{c} M \otimes \mathbb{C}$; причем если $l$ - минимальное число, обладающее этим свойством, то $M$ назьвается $l$-невырожденньм в точке $\xi$. В работе [23] П. Эбенфельт выписал нормальные формы уравнения 2 -невырожденной вешественно аналитической гиперповерхности в $\mathbb{C}^{3}$. В частности, он показал, что младшие члены уравнения гиперповерхности можно записать в одном из следуюших восьми вариантах. Если форма Леви поверхности $M$ в точке $\xi$ нулевая, т.е. оба собственных значения формы Леви равны нулю, то:

(A.1) $\operatorname{Im} w=\left|z_{1}\right|^{2}\left(z_{2}+\bar{z}_{2}\right)+r\left(z_{1}^{2} \bar{z}_{2}+\bar{z}_{1}^{2} z_{2}\right)+O\left(|z|^{4}+|\operatorname{Re} w||z|^{2}\right)$, где $r>0$;

(A.2) $\operatorname{Im} w=\left|z_{1}\right|^{2}\left(z_{2}+\bar{z}_{2}\right)+\left(z_{1}^{2} \bar{z}_{2}+\bar{z}_{1}^{2} z_{2}\right)+i\left|z_{1}\right|^{2}\left(z_{1}-\bar{z}_{1}\right)+O\left(|z|^{4}+|\operatorname{Re} w||z|^{2}\right)$;

(A.3) $\operatorname{Im} w=\left|z_{1}\right|^{2}\left(z_{2}+\bar{z}_{2}\right)+\left(z_{1} \bar{z}_{2}^{2}+\bar{z}_{1} z_{2}^{2}\right)+\left|z_{2}\right|^{2}\left(\lambda z_{2}+\bar{\lambda} \bar{z}_{2}\right)+O\left(|z|^{4}+|\operatorname{Re} w||z|^{2}\right)$, где $\lambda \in \mathbb{C}, \lambda \neq 0$;

(A.4) $\operatorname{Im} w=\left|z_{1}\right|^{2}\left(z_{1}+\bar{z}_{1}\right)+\left|z_{2}\right|^{2}\left(z_{2}+\bar{z}_{2}\right)+\left(\mu z_{1}^{2} \bar{z}_{2}+\overline{\mu z}_{1}^{2} z_{2}\right)+\left(\nu z_{1} \bar{z}_{2}^{2}+\overline{\nu z}_{1} z_{2}^{2}\right)+$ $O\left(|z|^{4}+|\operatorname{Re} w||z|^{2}\right)$, где $\mu, \nu \in \mathbb{C}, \mu \nu \neq 1$

(A.5) $\operatorname{Im} w=\left|z_{1}\right|^{2}\left(\eta z_{1}+\bar{\eta}_{1}\right)+\left(z_{1}^{2} \bar{z}_{2}+\bar{z}_{1}^{2} z_{2}\right)+\left(z_{1} \bar{z}_{2}^{2}+\bar{z}_{1} z_{2}^{2}\right)+O\left(|z|^{4}+|\operatorname{Re} w||z|^{2}\right)$, где $\eta \in \mathbb{C}$.

Если же форма Леви $M$ в точке $\xi$ имеет ровно одно ненулевое собственное значение, то:

(B.1) $\operatorname{Im} w=\left|z_{1}\right|^{2}+\left|z_{2}\right|^{2}\left(z_{2}+\bar{z}_{2}\right)+\gamma\left(z_{1}^{2} \bar{z}_{2}+\bar{z}_{1}^{2} z_{2}\right)+O\left(|z|^{4}+|\operatorname{Re} w||z|^{2}\right)$, где $\gamma=0,1$;

(B.2) $\operatorname{Im} w=\left|z_{1}\right|^{2}+\left(z_{1}^{2} \bar{z}_{2}+\bar{z}_{1}^{2} z_{2}\right)+O\left(|z|^{4}+|\operatorname{Re} w||z|^{2}\right)$;

(B.3) $\operatorname{Im} w=\left|z_{1}\right|^{2}+\left|z_{2}\right|^{2}\left(z_{1}+\bar{z}_{1}\right)+O\left(|z|^{4}+|\operatorname{Re} w||z|^{2}\right)$.

В работе [24] А. Ершова к каждому из восьми типов применила метод модельной поверхности. В частности, для каждого типа была получена точная оценка размерности группы автоморфизмов. Максимум размерности достигается для гиперповерхности $\operatorname{Im} w=\left|z_{1}\right|^{2}+\left|z_{2}\right|^{2}\left(z_{2}+\bar{z}_{2}\right)$ (это тип (В.1) при $\left.\gamma=0\right)$. Размерность всей групшы равна семи, размерность подгрупшы автоморфизмов, сохраняющих начало координат, трем.

Это вычисление, точнее та часть, которая касается типов (В.1), (В.2) и (В.3), позволяет закончить наше доказательство оценки размерности группы автоморфизмов произвольной неплоской вещественно аналитической гиперповерхности в $\mathbb{C}^{3}$. Действительно, если $M$ - вешественно аналитическая гиперповерхность в $\mathbb{C}^{3}$, не являюща- 
яся локально плоской, то множество точек $l$-невырожденности, где $l \leqslant 2$, есть открытое плотное подмножество $M[7]$. Повторяя наше рассуждение для произвольных 16 полей, получаем, что они линейно зависимы, т.е. $\operatorname{dim}$ Aut $M_{\xi} \leqslant 15$.

Итак, сформулированная вьше альтернатива справедлива и для поверхностей типа $(2,1)$.

Альтернатива (для гиперповерхности в $\mathbb{C}^{3}$ ):

либо $\operatorname{dim}$ Aut $M_{\xi}=\infty$ и $M$ в окрестности любой своей точки эквивалентна гиперплоскости;

либо $\operatorname{dim}$ Aut $M_{\xi} \leqslant \operatorname{dim}$ Aut $Q(2,1)=15$.

Таким образом, гиперквадрики являются самыми симметричньми гиперповерхностями и в $\mathbb{C}^{3}$.

$\mathrm{B} \mathbb{C}^{3}$ имеются две неэквивалентные гиперквадрики, но размерности их групп одинаковы и равны 15. Это специфика гиперповерхностей. Модельные поверхности более высокой коразмерности, например квадрики, могут иметь групшы различных размерностей, однако для каждого типа есть оценка. Потому для формулировки общей гипотезы обозначим через $D(n, k)$ и $d(n, k)$ максимумы размерностей групा автоморфизмов и подгрупп стабильности соответственно по всем модельным поверхностям типа $(n, k)$.

ГИПОТЕЗА о РАЗмЕРносТИ. Для ростка $M_{\xi}$ любого вещественно аналитического многообразия имеет место следующая альтернатива:

либо $\operatorname{dim}$ Aut $M_{\xi}=\infty$,

либо $\operatorname{dim}$ Aut $M_{\xi} \leqslant D(n, k)$ и $\operatorname{dim} \operatorname{Aut}_{\xi} M_{\xi} \leqslant d(n, k)$.

В заключение заметим, что если ставить задачу построения системы модельных поверхностей, охватьвающей все случаи вырождения, то такая задача естественно порождает древовидную классификацию ростков. Причем классификационное дерево не является конечным, что видно уже на примере гиперповерхностей в $\mathbb{C}^{2}$.

\section{СПИСОК ЛИТЕРАТУРЫ}

[1] А. В. Абросимов. Описание локально биголоморфных автоморфизмов стандартных квадрик коразмерности два // Матем. сб. 1993. Т. 184. № 10. С. 3-52.

[2] Е. Г. Анисова. Нуљ-квадрики коразмерности 4 в пространстве $\mathbb{C}^{7} / /$ Матем. заметки. 1997. T. 62. № 5. C. $657-665$.

[3] Е. Г. Анисова. Квадрики коразмерности 4 в $\mathbb{C}^{7}$ и их автоморфизмы // Матем. заметки. 1998. Т. 64. № 6. С. 803-811.

[4] А.Ф. Арбатский. О структуре группы нелинейных автоморфизмов $(3,3)$-квадрик // Матем. заметки. 1996. Т. 59. № 2. С. 164-173.

[5] M.S. Baouendi, P. Ebenfelt, L. P. Rothschild. Real Submanifolds in Complex Space and Their Mappings. Princeton, NJ: Princeton Univ. Press, 1999.

[6] M.S. Baouendi, P. Ebenfelt, L. P. Rothschild. Algebraicity of holomorphic mappings between real algebraic sets in $\mathbb{C}^{n} / /$ Acta Math. 1996. V. 177. P. 225-273.

[7] M.S. Baouendi, P. Ebenfelt, L.P. Rothschild. Local geometric properties of real submanifolds in complex space // Preprint Trita-Mat-1999-20. Stockholm: Royal Institute of Technology, 1999.

[8] M.S. Baouendi, X. Huang, L.P. Rothschild. Regularity of CR mappings between algebraic hypersurfaces // Invent. Math. 1996. V. 125. № 1. P. 13-36.

[9] M.S. Baouendi, L.P. Rothschild, D. Zaitsev. Equivalences of real submanifolds in complex space // Preprint, 2000, http://xxx.land.gov/abs/math.CV/0002186. 
[10] В. К. Белошапка. О размерности группы автоморфизмов аналитической гиперповерхности // Изв. АН СССР. Сер. матем. 1979. Т. 43. № 2. С. 243-266.

[11] В.К. Белошапка. Конечномерность группы автоморфизмов вещественно аналитической поверхности // Изв. АН СССР. Сер. матем. 1988. Т. 52. № 2. С. 437-442.

[12] В.К. Белошапка. О голоморфных преобразованиях квадрики // Матем. сб. 1991. T. 182. № 2. C. 203-219.

[13] В. К. Белошапка. Геометрические инварианты $C R$-многообразий // Матем. заметки. 1994. T. 55. № 5. C. 3-12.

[14] В. К. Белошапка. Инварианты $C R$-многообразий, связанные с касательной квадрикой // Матем. заметки. 1996. Т. 59. № 1. С. 42-52.

$[15]$ В. К. Белошапка. Локальные инварианты и запреты на отображения $C R$-многообразий // Матем. заметки. 1996. Т. 60. № 4. С. 588-592.

[16] В.К. Белошапка. Голоморфная классификация вещественных многообразий общего типа // Докл. РАН. 1998. Т. 360. № 4. С. 442-444.

[17] В. К. Белошапка. Кубическая модель вешественного многообразия // Матем. заметки. 2001. Т. 70. № 4. С. 503-519.

[18] В. К. Белошапка. Полиномиальные модели вещественных многообразий // Изв. РАН. Cер. матем. 2001. T. 65. № 4. C. 3-20.

[19] V.K. Beloshapka. CR-varieties of the type $(1,2)$ as varieties of "super-high" codimension // Russian J. Math. Phys. 1997. V. 5. № 3. P. 399-404.

[20] E. Cartan. Sur la géométrie pseudoconforme des hypersurfaces de deux variables complexes // Ann. Math. Pura Appl. (4). 1932. V. 11. P. 17-90. (Euvres. V. 2. P. 1231-1304.)

[21] S.S. Chern, J. K. Moser. Real hypersurfaces in complex manifold // Acta Math. 1974. V. 133. № 3-4. P. 219-271.

[22] Е. М. Чирка. Введение в геометрию $C R$-многообразий // УМН. 1991. Т. 46. № 1. C. $81-164$.

[23] P. Ebenfelt. Normal forms and biholomorphic equivalence of real hypersurfaces in $\mathbb{C}^{3} / /$ Indiana Univ. Math. J. 1998. V. 47. № 2. P. 311-366.

[24] А. Е. Ершова. Автоморфизмы 2-невырожденных гиперповерхностей в $\mathbb{C}^{3} / /$ Матем. заметки. 2001. Т. 69. № 2. С. 214-222.

[25] V. Ezhov, A. Isaev, G. Schmalz. Invariants of elliptic and hyperbolic CR-structures of codimension 2 // Internat. J. Math. 1999. V. 10. № 1. P. 1-52.

[26] V. Ezov, G. Schmalz. Holomorphic automorphisms of quadrics // Math. Z. 1994. V. 216. № 3. P. 453-470.

[27] V. Ezov, G. Schmalz. Poincaréautomorphisms for nondegenerate CR quadrics // Math. Ann. 1994. V. 298. № 1. P. 79-87.

[28] V. Ezov, G. Schmalz. A matrix Poincaré formula for holomorphic automorphisms of quadrics of higher codimension. Real associative quadrics // J. Geom. Anal. 1997. V. 8. № 1. P. 27-41.

[29] В. Ежов, Г. Шмальц. Автоморфизмы и голоморфные отображения стандартных CR-многообразий и области Зигеля // Прогресс в науке и технологии. Сер. Совр. матем. и ее прил. (Комплексньй анализ и теория представлений. Т. 1.). М.: ВИНИТИ (в печати).

[30] V. Ezov, G. Schmalz. Infinitesimale Starrheit hermitescher Quadriken in allgemeiner Lage // Math. Nachr. 1999. V. 204. P. 41-60.

[31] V. Ezov, G. Schmalz. Automorphisms of nondegenerate CR-quadrics and Siegel domains. Explicit description // Preprint, Max-Planck-Institute für Matematik, 1994; (to appear in J. Geom. Anal.).

[32] M. Freeman. Real submanifolds with degenerate Levi form // Proc. Sympos. Pure Math. 1977. V. 30. Part I. P. 141-147.

[33] T. Garrity, R. Mizner. Invariants of vector-valued bilinear and sesquilinear forms // Linear Algebra Appl. 1995. V. 218. P. 225-237.

[34] Г. С. Гиндикин, И. И. Пятецкий-Шапиро, Э.Б.Винберг. Классификация и каноническая реализация комплексных ограниченных однородных областей // Труды ММО. 1963. T. 12 . C. $404-437$. 
[35] Р. Хорн, Ч. Джонсон. Матричньй анализ. М.: Мир, 1989.

[36] M. Janet. Sur les systèmes d'équations aux dérivées partielles // J. Math. Pure Appl. 1920. V. 3. P. $65-151$.

[37] А.С. Лабовский. О размерности группы биголоморфных автоморфизмов вещественно аналитических поверхностей // Матем. заметки. 1997. Т. 61. №3. С. 349-358.

[38] Л. Д. Ландау, Е. М. Лифбшиц. Квантовая механика. М.: Наука, 1972.

[39] А. В. Лобода. Порождающие вещественно аналитические многообразия коразмерности 2 в $\mathbb{C}^{2}$ и их биголоморфные отображения // Изв. АН СССР. Сер. матем. 1988. Т. 52. № 5. С. $970-990$.

[40] А. В. Лобода. Однородные вещественные гиперповерхности в $\mathbb{C}^{3}$ с двумерными группами изотропии // Труды МИАН. 2001. Т. 235. С. 114-142.

[41] R. Mizner. CR structures of codimension 2 // J. Differential Geom. 1989. V. 30. № 1. P. $167-190$.

[42] I. Naruki. Holomorphic extention problem for standard real submanifolds of second kind // Publ. Res. Inst. Math. Sci. 1970. V. 6. №1. P. 113-187.

[43] Н.Ф. Палинчак. О квадриках высокой коразмерности // Матем. заметки. 1994. Т. 55. № 5. C. $110-115$.

[44] Н.Ф. Палинчак. Вещественные квадрики коразмерности три в $\mathbb{C}^{6}$ и их нелинейные автоморфизмы // Изв. РАН. Сер. матем. 1995. Т. 59. № 3. С. 159-179.

[45] Н.Ф. Палинчак. О с-жестких квадриках // Деп. в ВИНИТИ РАН 10.04.95, № 973-В95.

[46] С.И. Пинчук. О голоморфных отображениях вещественно аналитических гиперповерхностей // Матем. сб. 1978. Т. 105. С. 574-593.

[47] H. Poincaré. Les fonctions analytiques de deux variables et la représentation conforme // Rend. Circ. Mat. Palermo. 1907. V. 23. P. 185-220.

[48] И.И. Пятецкий-Шапиро. Геометрия классических областей и теория автоморфных функций. М.: Физматгиз, 1961.

[49] А. Рябоненко. О жесткости кубики типа $\left(n, n^{2}+1\right) / /$ Дипломная работа. М.: механико-математический ф-т МГУ, 2001.

[50] I. Satake. Algebraic Structures of Symmetric Domains. Tokyo / Princeton: Ivanami Shoten / Princeton Univ. Press, 1980. (Kâno Memorial Lectures. V. 4.)

[51] Е. Н. Шананина. Модели $C R$-многообразий типа $(1, k)$ при $3 \leqslant k \leqslant 7$ и их автоморфизмы // Матем. заметки. 2000. Т. 67. № 3. С. 452-459.

[52] С.Н. Шевченко. Описание алгебры инфинитезимальных автоморфизмов квадрик коразмерности два и их классификация // Матем. заметки. 1994. Т. 55. № 5. С. 142-153.

[53] С.Н. Шевченко. Квадрики коразмерности два и их автоморфизмы // Изв. РАН. Сер. матем. 1994. Т. 58. № 4. C. 149-172.

[54] N. K. Stanton. Infinitesimal CR automorphisms of rigid hypersurfaces // Amer. J. Math. 1995. V. 117. № 1. P. 141-167; 1996. V. 118. №1. P. 209-233.

[55] С. Стернберг. Лекции по дифференциальной геометрии. М.: Мир, 1970.

[56] A. Sukhov. Segre varieties and Lie symmetries // Publ. Inst. Rech. Math. Av., Lille. V. 50, 1999.

[57] A. Sukhov. On CR-mappings of real quadric manifolds // Michigan Math. J. 1999. V. 41. P. $143-150$.

[58] N. Tanaka. On the pseudo-conformal geometry of hypersurfaces of the space of $n$ complex variables // J. Math. Soc. Japan. 1962. V. 14. P. 397-429; II. Graded Lie algebras and geometric structures // Proc. Unated States-Japan Seminar in Differential Geometry (Kyoto, 1965), 1966. P. 147-150.

[59] A. Tress. Détermination des invariants ponctuels del'équation différentielle du second ordre $y^{\prime \prime}=\omega\left(x, y, y^{\prime}\right)$. Leipzig: Hirzel, 1896.

$[60]$ А. Е. Туманов. Конечномерность группы $C R$-автоморфизмов стандартного $C R$-многообразия и собственные голоморфные отображения областей Зигеля // Изв. АН СССР. Сер. матем. 1988. Т. 52. № 3. С. 651-659.

[61] А.Е. Туманов. Геометрия $C R$-многообразий // Итоги науки и техники. Совр. пробл. матем. Фундам. напр. Т. 9. М.: ВИНИТИ, 1986. С. 225-245. 
[62] H. W. Turnbull. On the equivalence of pencils of Hermitian forms // Proc. London Math. Soc. (2). 1935. T. 39. №3. C. 232-248.

[63] П. Б. Уткин. Гипотеза о размерности для квадрик коразмерности три и более // Матем. заметки (в печати).

[64] А. Г. Витушкин. Голоморфные отображения и геометрия поверхностей // Итоги науки и техники. Совр. пробл. матем. Фундам. напр. Т. 7. М.: ВИНИТИ, 1985. С. 167-226.

[65] S. Webster. On the mappings problem for algebraic real hypersurfaces // Invent. Math. 1977. V. 43. P. 53-68.

[66] D. Zaitsev. Regularization of birational group operations in sense of Weil // J. Lie Theory. 1995. V. 5. № 2. P. 207-224.

Московский государственный

Поступила в редакцию

университет им. М. В. Ломоносова

03.12.2001

E-mail: vkb@strogino.ru 